\title{
Fast H3K9 methylation promoted by CXCL12 contributes to nuclear changes and invasiveness of T-acute lymphoblastic leukemia cells
}

\author{
Elena Madrazo ${ }^{1,5}$, Raquel González-Novo (iD ${ }^{1,5}$, Cándido Ortiz-Placín (iD ${ }^{1}$, Mario García de Lacoba (iD) ${ }^{2}$, África González-Murillo (iD ${ }^{3,4}$, \\ Manuel Ramírez $z^{3,4}$ and Javier Redondo-Muñoz (iD ${ }^{1 凶}$
}

(c) The Author(s), under exclusive licence to Springer Nature Limited 2022

T-acute lymphoblastic leukemia (T-ALL) is an aggressive hematological malignancy that comprises the accumulation of malignant T-cells. Despite current therapies, failure to conventional treatments and relapse are frequent in children with T-ALL. It is known that the chemokine CXCL12 modulates leukemia survival and dissemination; however, our understanding of molecular mechanisms used by T-ALL cells to infiltrate and respond to leukemia cells-microenvironment interactions is still vague. In the present study, we showed that CXCL12 promoted H3K9 methylation in cell lines and primary T-ALL cells within minutes. We thus identified that CXCL12-mediated H3K9 methylation affected the global chromatin configuration and the nuclear mechanics of T-ALL cells. Importantly, we characterized changes in the genomic profile of T-ALL cells associated with rapid CXCL12 stimulation. We showed that blocking CXCR4 and protein kinase C (PKC) impaired the H3K9 methylation induced by CXCL12 in T-ALL cells. Finally, blocking H3K9 methyltransferases reduced the efficiency of T-ALL cells to deform their nuclei, migrate across confined spaces, and home to spleen and bone marrow in vivo models. Together, our data show novel functions for CXL12 as a master regulator of nuclear deformability and epigenetic changes in T-ALL cells, and its potential as a promising pharmacological target against T-ALL dissemination.

Oncogene; https://doi.org/10.1038/s41388-021-02168-8

\section{INTRODUCTION}

Acute lymphoblastic leukemia (ALL) is an aggressive hematological disorder that represents the most common childhood cancer. ALL can be divided into B-ALL (80\%) and T-ALL (20\%) according to the malignant transformation of B- and T-cell precursors [1]. ALL dissemination remains a major clinical problem, as ALL cells respond to different microenvironmental signals and infiltrate various organs, including the spleen, lymph nodes, liver, and central nervous system $[2,3]$. ALL resistance associates with these specific tissues where leukemia cells resist therapies. Thus, defining novel molecular mechanisms used by ALL cells is essential to target leukemia dissemination and reduce side effects of systemic treatment in patients.

Chemokines are chemotactic cytokines that regulate many cellular functions, including migration, survival, proliferation, and activation [4]. CXCL12 (CXC motif chemokine 12)/Stromal cellderived factor-1 (SDF-1a) is a chemokine produced by several cell types (including CXCL12-abundant reticular/CAR, endothelial and stromal cells) that is critical for steady-state conditions of leukocytes and the homing of hematopoietic stem cells into the bone marrow (BM) [5]. In T-ALL cells, CXCL12 is critical for cells escaping from the thymus into the $\mathrm{BM}$, for leukemia initiating cells, and for transendothelial migration and in vivo dissemination of T-ALL cells [6-8]. Studies blocking CXCL12 and its receptor CXCR4 have shown the potential effect of targeting this chemokine on leukemia maintenance and progression [9, 10]; however, the molecular implications of CXCL12 on leukemia cell biology remains intriguing and should be elucidated to maximize its potential as a therapeutic target in T-ALL.

Chromatin structure is regulated by multiple epigenetic alterations, including the methylation of histones, of which a key chromatin mark is the histone 3 lysine 9 (H3K9) methylation. [11]. Emerging evidence indicates that deformability and mechanical changes of the nucleus are crucial for cancer cell migration [12]. This nuclear deformability is regulated mainly by the expression of nuclear lamins and the chromatin structure $[13,14]$. However, the molecular mechanisms by which CXCL12 might control H3K9 methylation and nuclear changes during T-ALL cell migration have not been reported.

In the present work, we demonstrated that CXCL12 induced H3K9 methylation in both cell lines and primary pediatric T-ALL cells. We described that H3K9 methylation induced by CXCL12 promoted global chromatin structure modification, which correlated with changes in the mechanical properties of the nucleus

\footnotetext{
${ }^{1}$ Department of Molecular Medicine, Centro de Investigaciones Biológicas Margarita Salas (CIB Margarita Salas-CSIC), Madrid, Spain. ${ }^{2}$ Bioinformatics and Biostatistics Unit, Centro de Investigaciones Biológicas Margarita Salas (CIB Margarita Salas-CSIC), Madrid, Spain. ${ }^{3}$ Department of Paediatric Haematology \& Oncology, Hospital Universitario Niño Jesús, Madrid, Spain. ${ }^{4}$ Health Research Institute La Princesa, Madrid, Spain. ${ }^{5}$ These authors contributed equally: Elena Madrazo, Raquel González-Novo.

凶email: Javier.redondo@cib.csic.es
} 
and the transcriptional profile of T-ALL cells. We showed that protein kinase $C$ (PKC) inhibition impaired the CXCL12-mediated H3K9 methylation in T-ALL cells. Furthermore, targeting or blocking the enzymes involved in H3K9 methylation abrogated the migration and squeezing of T-ALL cells in vitro and in vivo systems. Taken together, our findings identified a critical role of CXCL12 on nuclear changes of T-ALL cells and suggested epigenetic changes induced by this chemokine as possible therapeutic targets to block T-ALL migration.

\section{MATERIAL AND METHODS Primary samples}

Samples were obtained from Dr. Ramírez (Hospital Universitario Infantil Niño Jesús) with informed consent for research purposes, and the procedures were approved by the CSIC Ethics Committee and the Institutional Review Board of the Hospital Universitario Niño Jesús (R0070/15). A total of ten ALL patients under 14 years old were retrospectively included in this study. ALL diagnosis and treatment were defined according to SEHOP-Pethema 2013 (Spanish Program for the Treatment of Hematologic Diseases). Patients' characteristics are provided in Supplementary Table S1.

\section{Cells}

The human T-ALL cell lines (Jurkat and CCRF-CEM) and the B-ALL cell line (Reh). Cell lines and primary cells were cultured in RPMI 1640 with L-glutamine and $\mathrm{NaHCO}_{3}$ (Sigma-Aldrich) and $10 \%$ fetal bovine serum (Sigma-Aldrich), in $5 \% \mathrm{CO}_{2}$ at $37^{\circ} \mathrm{C}$. Cells were routinely tested for mycoplasma contamination (Mycoplasma Gel Detection Kit, Biotools).

\section{Cellular fractionation}

Cells were lysed with buffer A (HEPES [pH 8.0] $10 \mathrm{mM}, \mathrm{KCl} 10 \mathrm{mM}, \mathrm{MgCl}_{2}$ $1.5 \mathrm{mM}$, sucrose $0.34 \mathrm{M}$, glycerol $10 \%$, DTT $1 \mathrm{mM}, \mathrm{Tx}-1000.1 \%$ ) for $5 \mathrm{~min}$ at $4{ }^{\circ} \mathrm{C}$. Then, cytoplasmic fractions were collected from centrifugation at $3200 \mathrm{rpm}$ for $5 \mathrm{~min}$. The pellet was washed once with buffer $A$ and resuspended in RIPA buffer (NaCl $1 \mathrm{M}, \mathrm{NP}-401 \%$, Sodium deoxycholate $0.5 \%$, SDS $0.1 \%$, Tris- $\mathrm{HCl}$ [pH 7.4] $50 \mathrm{mM}$ ) with protease inhibitors. After $15 \mathrm{~min}$, soluble nuclear fractions were collected from centrifugation $(5 \mathrm{~min}$, $\left.4{ }^{\circ} \mathrm{C}, 13,500 \mathrm{rpm}\right)$ and chromatin bound fractions were resuspended in sample buffer (VWR) and sonicated.

\section{In vivo short-term leukemia homing assay}

NOD.Cg-Prkdcscid $112 \mathrm{rgtm} 1 \mathrm{Wj} / \mathrm{SzJ}$ (NSG) mice were purchased from Charles River Laboratories and bred and maintained at the Servicio del Animalario del Centro de Investigaciones Energéticas, Medioambientales y Tecnológicas (CIEMAT) with registro 28079-21 A. All mice were used following guidelines issued by the European and Spanish legislation for laboratory animal care. All experiments involving animals were approved by the OEBA (Organ for Evaluating Animal Wellbeing) at CIEMAT and Madrid Regional Department of Environment, with reference PROEX 185/ 15. $2.5 \times 106$ control and BIX-01294 or chaetocin inhibited Jurkat cells were labeled with CFSE $5 \mu \mathrm{M}$ or Far Red Cell Tracker $1 \mu \mathrm{M}$ for 30 min in RPMI. Control and inhibited cells were mixed 1:1 and intravenously (IV) administrated into 10-12 weeks-old NSG male animals to compare the cell migration. Sacrifice was performed $3 \mathrm{~h}$ after inoculation. BMs from femurs and spleens were extracted, processed through mechanical disaggregation, and stained cells were resuspended in the PBS for flow cytometry (FACSCantoTM II, Becton Dickinson). Samples were acquired in a FacsCanto II (BD) cytometer and the number of Far Red cell tracker or CFSE positive cells analyzed using FacsDiva software.

\section{RESULTS}

CXCL12 promotes a fast H3K9 methylation in T-ALL cells

First, we analyzed H3K9me2/3 levels in primary samples from pediatric patients with ALL (Supplementary Table S1) and observed that $15 \mathrm{~min}$ of stimulation with CXCL12 upregulated the levels of $\mathrm{H} 3 \mathrm{~K} 9 \mathrm{me} 2 / 3$ in T-ALL cells compared to untreated conditions (Fig. 1a). Interestingly, B-ALL cells downregulated H3K9me2/3 levels compared to control (unstimulated) conditions. We visualized $\mathrm{H} 3 \mathrm{~K} 9 \mathrm{me} 2 / 3$ signal in ALL cell lines seeded on poly-lysine and found that
CXCL12 stimulation increased the H3K9 methylation only in T-ALL (Jurkat and CCRF-CEM) but not in the B-ALL (Reh) cell lines (Fig. 1b, c). CXCL12 is mainly produced by resident cells in the BM microenvironment and its immobilization is fundamental for cell adhesion [15], we cultured ALL cells in the presence of soluble or immobilized CXCL12 and confirmed that both stimuli promoted H3K9 methylation in Jurkat and CCRF-CEM cells (Fig. 1d and Supplementary Fig. S1a). Furthermore, we confirmed that Reh cells did not upregulate H3K9 methylation in response to soluble or immobilized CXCL12 (Supplementary Fig. S1b). To explore whether this epigenetic change induced by CXCL12 only in T-ALL cells was reversible, Jurkat and CCRF-CEM cells were stimulated with CXCL12, washed, and cultured in fresh media without chemokine for an additional hour. CXCL12 stimulation increased the levels of H3K9me2/3 in both T-ALL cell lines within minutes, and these cells regained the basal level of H3K9me3 in the absence of the chemokine (Supplementary Fig. S1c, d). We also identified that H3K9 methylation induced by CXCL12 was associated with a reduction in the nuclear area (Supplementary Fig. S1e), suggesting that epigenetic changes induced by CXCL12 might regulate the nuclear morphology of ALL cells. Although no differences were found in the cell size distributions upon CXCL12 stimulation by flow cytometry (Fig. 1e); we observed that isolated nuclei from CXCL12-stimulated T-ALL cells were smaller than those from control cells (Fig. 1f). Furthermore, this only occurred in T- and not in B-ALL cells (Supplementary Fig. S1f). Together, our results indicated that $\mathrm{CXCL} 12$ promoted a fast and transient upregulation of $\mathrm{H} 3 \mathrm{~K} 9 \mathrm{me} 2 / 3$ levels in T-ALL cells.

\section{Fast epigenetic changes induced by $C X C L 12$ regulate the genomic landscape of ALL cells}

To investigate how H3K9 methylation induced by CXCL12 was associated with changes in the genomic landscape of T-ALL, we performed a chromatin immunoprecipitation assay (ChIP-seq) from control or CXCL12-stimulated Jurkat cells. We observed an upregulation of $\mathrm{H} 3 \mathrm{~K} 9 \mathrm{me} 3$ peaks on the whole genome of Jurkat cells stimulated with CXCL12 compared to control cells (Fig. 2a) and mapped a total of 1739 differentially regulated $\mathrm{H} 3 \mathrm{~K} 9 \mathrm{me} 3$ peaks, 1185 of which were upregulated upon CXCL12 stimulation (Fig. 2b). Overall, when we quantified the genomic location annotation of these $\mathrm{H} 3 \mathrm{~K} 9 \mathrm{me} 3$ peaks, we found that most of them were located mainly at protein-coding regions belonging to genes or transcripts (Supplementary Fig. S2a, b). By focusing on these protein-coding regions, we observed that the biggest difference by K-means clustering corresponded to 45 regions (Supplementary Fig. S2c). GO-term enrichment showed differences in genes related to protein deglutamylathion, polarity and membranerelated signaling, calcium signaling, exocytosis among others (Supplementary Fig. S2d, Supplementary Table S2). To further identify the potential effects of CXCL12 stimulation on the transcriptional activity of T-ALL cells, we performed a whole transcriptome analysis of Jurkat cells stimulated with CXCL12. Consistent with the upregulation of $\mathrm{H} 3 \mathrm{~K} 9 \mathrm{me} 3$ peaks, cells treated with CXCL12 showed that 149 and 212 genes to be significantly up- and downregulated, respectively (Fig. 2c and Supplementary Table S3), indicating that CXCL12 stimulation might repress transcription at short times. Among the top 20 most enriched GO terms were related to Ras activity, cation channels, calcium signaling, cell adhesion, and cell receptor signaling (Fig. $2 \mathrm{~d}$ and Supplementary Table S4). Furthermore, gene set expression analysis (GSEA) showed a small set of genes altered in ChIP-seq and microarray analyses (Supplementary Table S5), without any correlation between $\mathrm{H} 3 \mathrm{~K} 9 \mathrm{me} 3$ peaks resolved by ChIP-seq and the transcriptional analysis (Fig. 2e and Supplementary Fig. S2e). Together, these results indicate that $\mathrm{CXCL} 12$ promotes higher H3K9me3 occupancy at the whole genome, without significant changes at the transcriptional stage of ALL, suggesting that the relevance of this epigenetic change might be at a biomechanical level. 
a

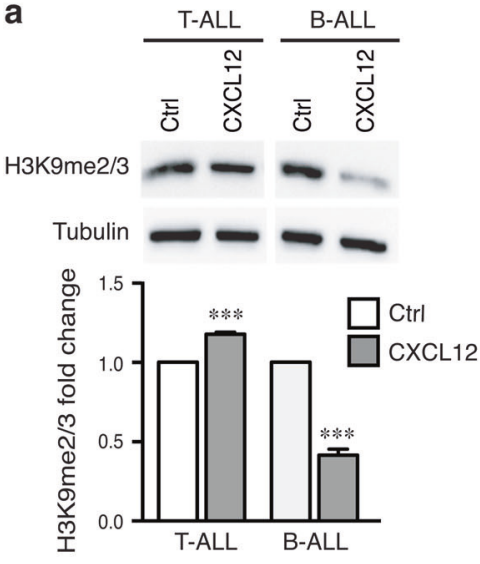

c

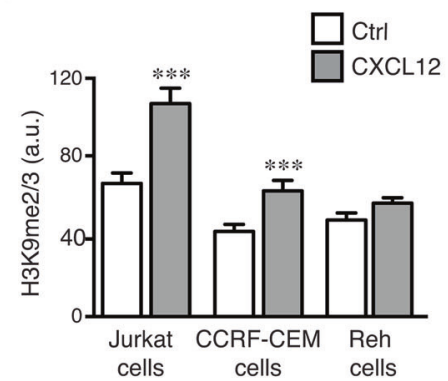

e

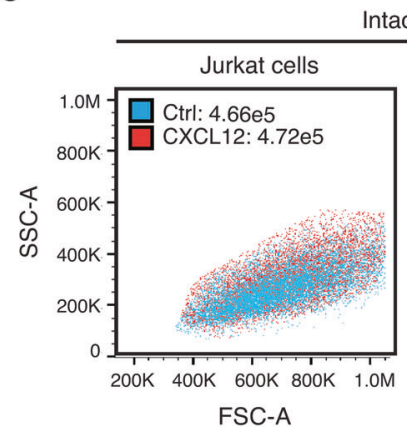

Intact cells

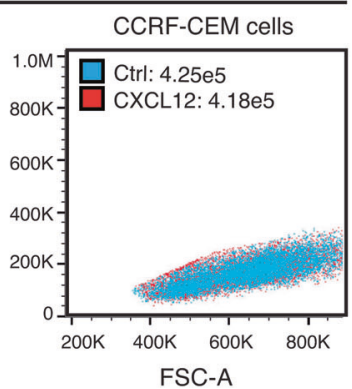

d b
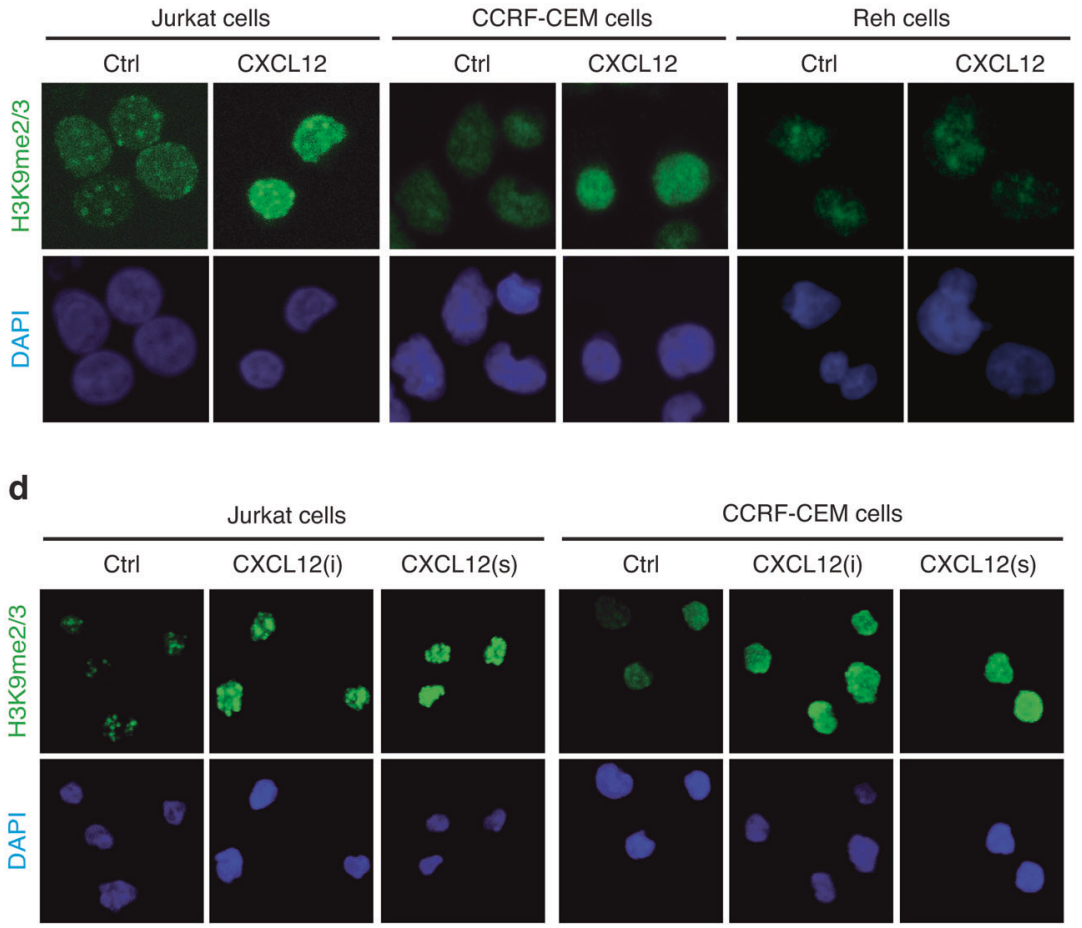

CCRF-CEM cells

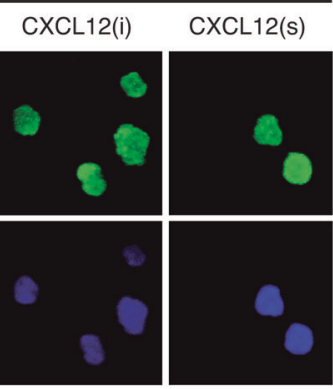

f

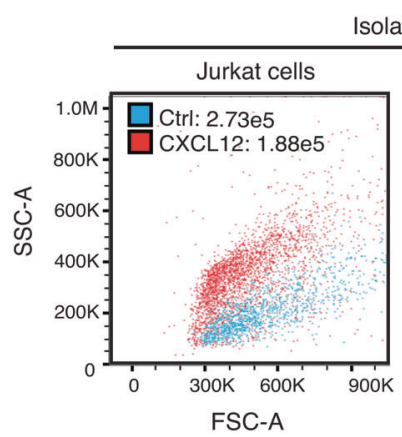

solated nuclei

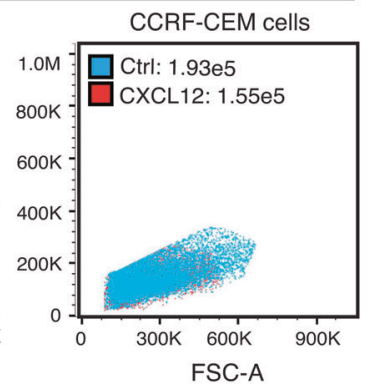

Fig. 1 CXCL12 promotes H3K9 methylation in T-ALL cells. a Primary ALL cells from patients were incubated for 15 min with CXCL12 (100 ng/ $\mathrm{ml}$ ). Subsequently, cells were lysed for detection of H3K9me2/3 levels by western blotting. Mean $n=3 \pm$ SEM. b T- ALL (Jurkat and CCRF-CEM) and B-ALL (Reh) cell lines were seeded on poly-lysine coated coverslips and stimulated with CXCL12 (100 ng/ml) for $15 \mathrm{~min}$. Then, cells were fixed, permeabilized and stained for the indicated markers. Confocal images show representative images of H3K9 methylation induced by CXCL12 in Jurkat and CCRF-CEM cells. c Graph shows the mean intensity (arbitrary units, a.u.) of H3K9me2/3 of ALL cell lines in (b). Mean $n=$ 60 cells \pm SEM. d Jurkat and CCRF-CEM cells were seeded on poly-lysine coated coverslips in the absence or presence of immobilized (i) or soluble (s) CXCL12 for $15 \mathrm{~min}$. Then, cells were fixed, permeabilized and stained for the indicated markers. e Jurkat and CCRF-CEM cells stimulated or not with CXCL12 for $15 \mathrm{~min}$ were analyzed by flow cytometry. The plot represents volume distributions of Ctrl (blue) and CXCL12-stimulated (red) cells measured by FSC-A and SSC-A. Values indicate the geometric mean. $f$ Isolated nuclei from Jurkat and CCRF-CEM cells stimulated or not with CXCL12 for 15 min were analyzed by flow cytometry. The plot represents the volume distribution of Ctrl (blue) and CXCL12-stimulated (red) isolated nuclei measured by FSC-A and SSC-A. Values indicate the geometric mean. $P$-values are indicated by asterisks $* * * P<0.001$

\section{H3K9 methylation induced by CXCL12 alters the global chromatin conformation of ALL cells}

As H3K9 methylation is a canonical marker for heterochromatin [16], we determined the level of chromatin compaction induced by CXCL12 in ALL cells. By using a DNAse I sensitivity assay [17], we observed that only T-ALL cells presented more resistance to DNA digestion upon CXCL12 stimulation (Fig. 3a, b). Then, we carried out in situ DNAse digestion of ALL cells upon CXCL12 stimulation to validate our findings. We found that CXCL12 induced the compaction and more resistant chromatin to DNAse I digestion in T- (Fig. 3c), but not in B-ALL cells (Supplementary Fig. S3a). Based on these results, we additionally measured the sensitivity of ALL cells to micrococcal nuclease (MNase), an enzyme that digests the nucleosome linker regions. We confirmed that CXCL12 stimulation also promoted a more compacted chromatin resistant to MNAse digestion in T-ALL cell lines (Fig. 3d, e). Chromatin compaction takes place during cell cycle progression, and it has been reported that CXCL12 promote cell proliferation [18]. As H3K9 methylation occurred within minutes, we speculated that it should not interfere with other prosurvival effects induced by this chemokine. To test this hypothesis, we evaluated the proliferation upon CXCL12 addition. As expected, CXCL12 stimulation increased slightly the proliferation ratio of all ALL cell lines tested (Supplementary Fig. S3b); 
a

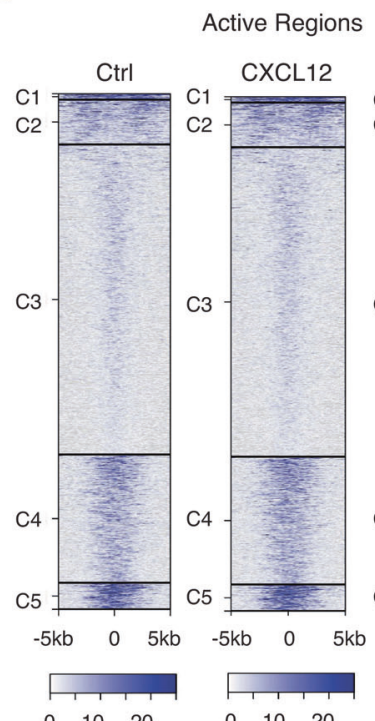

C

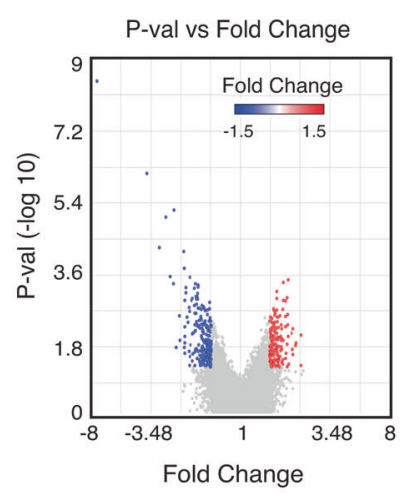

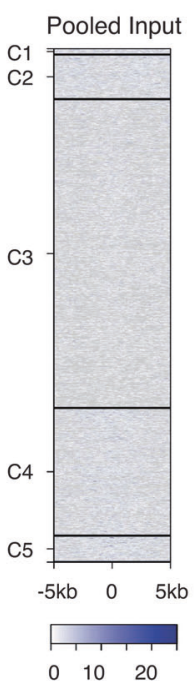

d
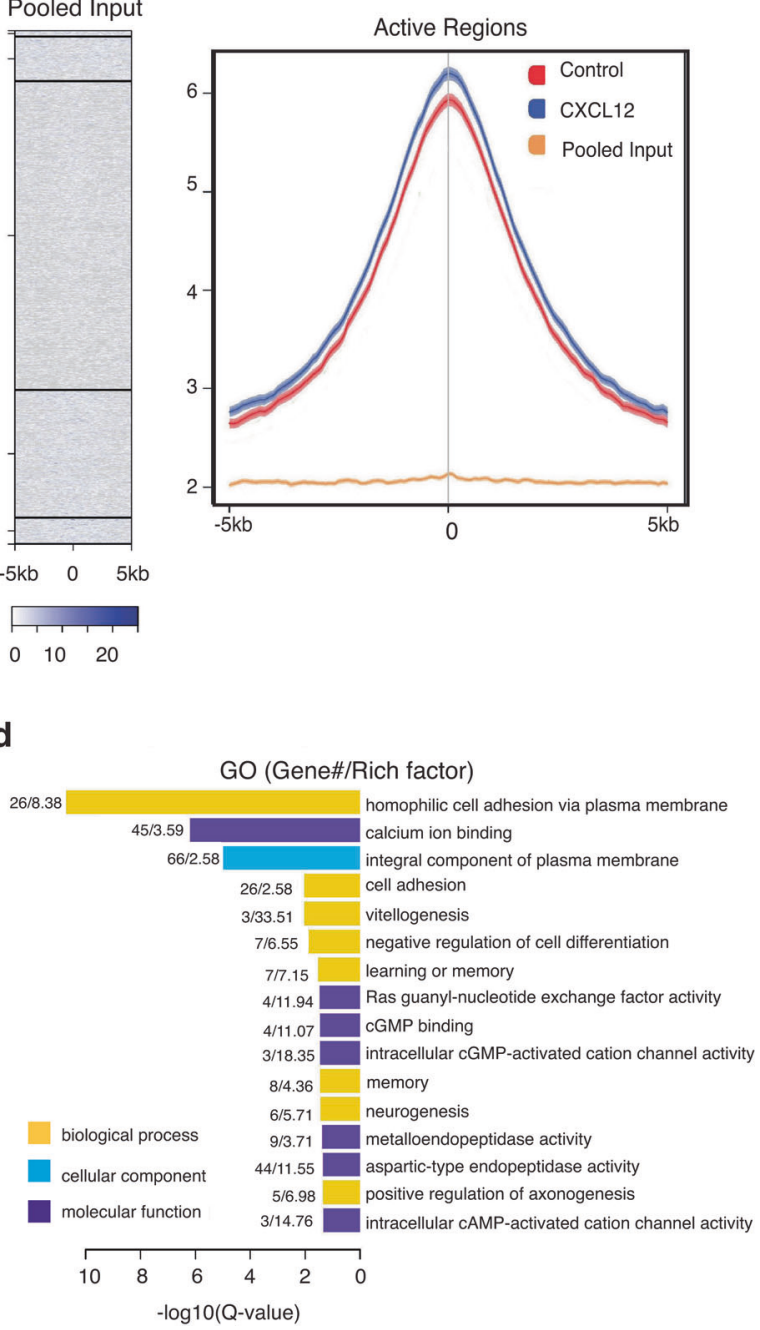

b

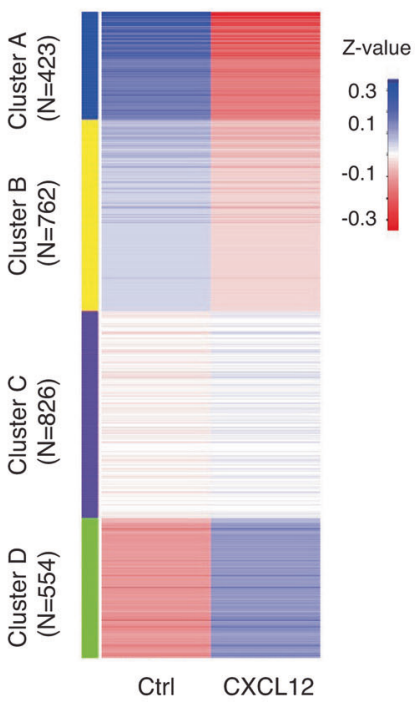

e

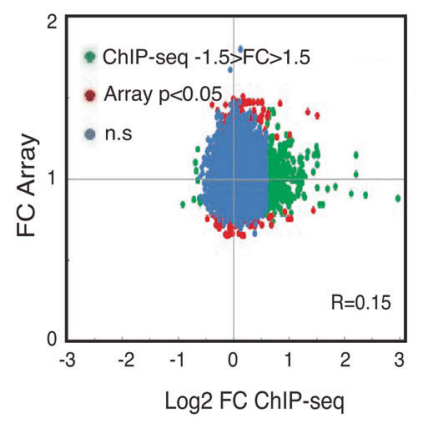

Fig. 2 CXCL12 alters the epigenetic and transcriptional landscape of T-ALL cells. a Jurkat cells were cultured in medium (Ctrl) or with CXCL12 $(100 \mathrm{ng} / \mathrm{ml})$ for $15 \mathrm{~min}$, and H3K9 methylation changes were analyzed by ChIP-seq. Heatmaps for distance-base clustering of differential H3K9me3 tags across the whole genome and plots for their average values. $\mathbf{b}$ K-means clustering of genomic-wide changes relating to the H3K9me3 epigenetic signal induced by CXCL12. c Volcano-plot shows the differential expression of genes between control cells and stimulated cells by CXCL12 analyzed by microarray experiments. Red and blue dots are denoting up- and downregulated genes, respectively. d Jurkat cells were cultured without (Ctrl) with CXCL12 $(100 \mathrm{ng} / \mathrm{ml})$ for $15 \mathrm{~min}$, and transcriptional changes were analyzed by microarray. Graph shows the GO-term analysis of altered genes in Jurkat cells upon CXCL12 stimulation from microarray experiments. e Correlation plot and quantification of H3K9me3 peaks resolved by ChIP-seq and differential gene expression obtained from the microarray.

although no significant differences were found in the $S$ phase entry and G2 progression (Supplementary Fig. S3c). Together, these results suggest that CXCL12 stimulation was able to successfully induce a global condensation of chromatin in T-ALL cells.

\section{CXCL12 stimulation induces a different mechanical behavior of the nucleus}

Previous analyses have shown that chromatin changes contribute to the mechanical response of the cell nucleus $[19,20]$. The chromatin compaction induced by CXCL12 in T-ALL cells suggested that the chromatin might be relevant for the mechanical response of leukemia cells. Consistent with this hypothesis, we tested a cell confiner developed to induce physical stress [21]. By using confocal stacks and reconstructed 3D renderings, we examined the area of isolated nuclei from control or CXCL12-stimulated Jurkat cells seeded on confinement plates of defined height $(3 \mu \mathrm{m})$ (Fig. $4 \mathrm{a}, \mathrm{b})$. Under physical forces, isolated nuclei from control Jurkat cells showed a nuclear deformability ratio of 1.39, whilst those nuclei from cells upon CXCL12 stimulation revealed a less prominent ratio (Fig. 4c). We observed a similar effect on isolated nuclei of CCRF-CEM cells upon CXCL12 stimulation (Supplementary Fig. S4a, b), whilst Reh cells showed no differences in the nuclear deformability ratio of control and CXCL12-treated conditions (Supplementary Fig. S4c, d). Based on these findings, we measured the morphology of isolated nuclei from CXCL12-stimulated ALL cells under osmotic stress. Adding EDTA promoted more prominent nuclear swelling in control conditions than in isolated nuclei from CXCL12stimulated cells. Consistent with the mechanical compression and nuclear compaction, we observed this phenomenon in T- but not in B-ALL cells (Fig. 4d, e). Together, these results revealed that 
a

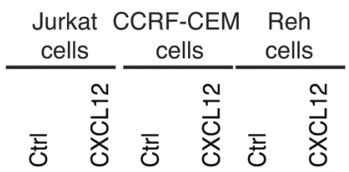

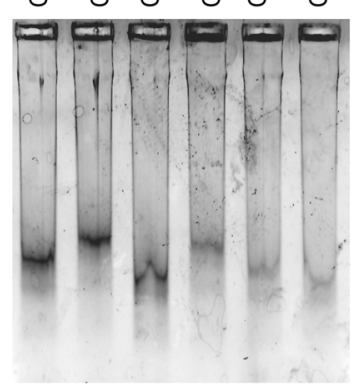

C
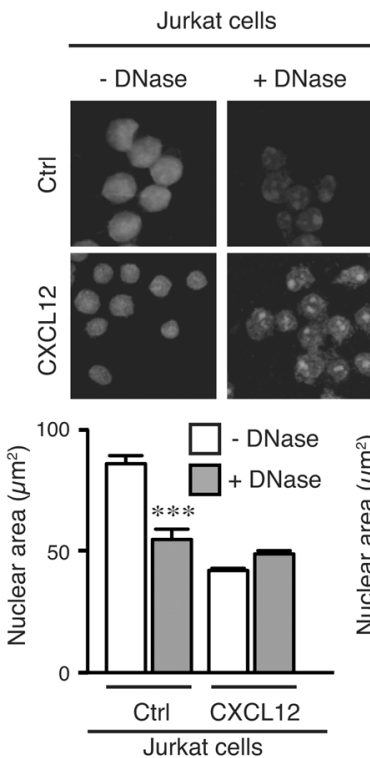

b
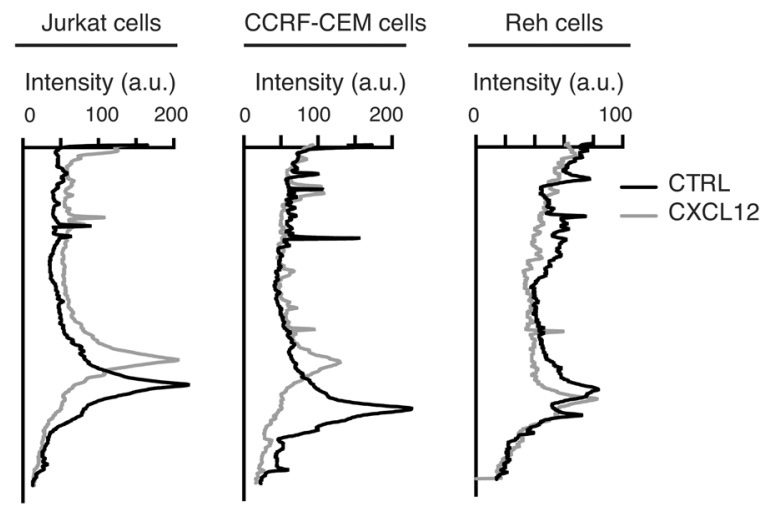

d

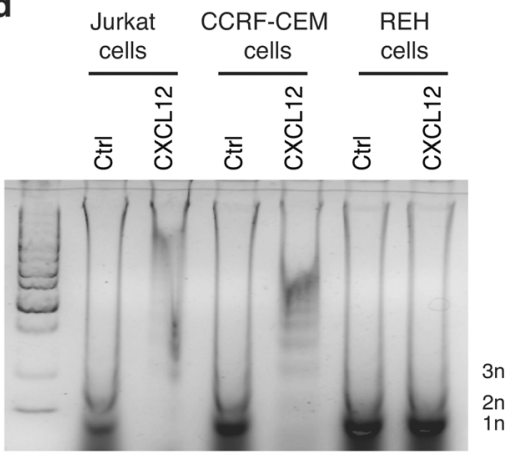

e

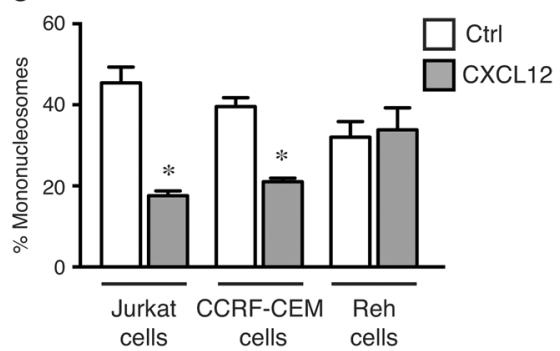

Fig. 3 CXCL12 induces global chromatin compaction in T-ALL cells. a Jurkat, CCRF-CEM and Reh cells were in fresh medium (Ctrl) or with CXCL12 (100 ng/ml) for $15 \mathrm{~min}$. Then, cells were collected, lysed and DNA was digested with DNAse I for 15 min. Gel image shows chromatin degradation upon DNAse activity. b Graph shows the digested DNA profiles. Black line indicates control (Ctrl) conditions and gray line indicates upon CXCL12 stimulation. c Jurkat and CCRF-CEM cells were seeded on poly-lysine coated coverslips, and cultured in fresh medium (Ctrl) or stimulated with CXCL12 $(100 \mathrm{ng} / \mathrm{ml})$ for $15 \mathrm{~min}$. Then, cells were subjected to in situ DNase I digestion, fixed, and stained with DAPI. Graph shows the nuclear projected area. Mean $\mathrm{n}=30 \pm$ SEM. d Jurkat, CCRF-CEM and Reh cells were in fresh medium (Ctrl) or with CXCL12 $(100 \mathrm{ng} / \mathrm{ml})$ for $15 \mathrm{~min}$. Cells were lysed, and chromatin was digested by microccocal DNAse (MNase) for 15 min. Nucleosome releasing was resolved on $2 \%$ agarose gels. Lines indicate mono- $(1 n)$, di- $(2 n)$ and tri- $(3 n)$ nucleosomes upon MNAse digestion. e Graph shows the percentage of released mononucleosomes upon MNAse digestion. Mean $n=3 \pm$ SEM. $P$-values are indicated by asterisks ${ }^{* * *} P<0.001$.

chromatin changes induced by CXCL12 had a direct impact on the mechanical properties and the deformability of the nucleus of T-ALL cells.

\section{PKC activity regulates the $\mathrm{H} 3 \mathrm{~K} 9$ methylation induced by CXCL12 in T-ALL cells}

There are two cell receptors for CXCL12, CXCR4, and CXCR7, and both have relevance for ALL cell migration and invasiveness $[22,23]$. As only T-ALL cells upregulated H3K9 methylation in response to CXCL12, we treated Jurkat with AMD3100 and CCX771 (CXCR4 and CXCR7 inhibitors, respectively) and observed that only CXCR4 inhibition abrogated the effect of CXCL12 on H3K9me2/3 levels (Fig. 5a, b). Similar results were observed in CCRF-CEM cells (Supplementary Fig. S5a, b). It is well known that CXCR4/CXCL12 axis promotes cell migration through multiple signaling pathways, including PKC activation [24]. We determined that inhibitors against conventional PKC prevented the H3K9 methylation induced by CXCL12 in T-ALL cell lines (Fig. 5c). By using Phorbol 12-myristate 13-acetate (PMA), we observed that PKC activation induced upregulation of $\mathrm{H} 3 \mathrm{~K} 9$ methylation in both T- (Fig. $5 \mathrm{~d}$, e) and, interestingly, also in B-ALL cells (Supplementary Fig. S5c). We examined the nuclear pattern of conventional PKC localization in ALL cells and found that PKC $\beta$ was expressed both in the cytoplasm and the nucleus of ALL cells, whilst most PKCa showed a cytoplasmic localization (Supplementary Fig. S5d). To confirm that this nuclear localization of PKC $\beta$ was not a microscopy artifact, we analyzed the subcellular localization of PKC in T- and B-ALL cells and confirmed that PKCa was detected primarily in the 
a
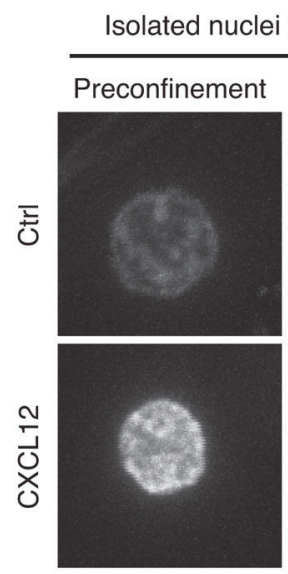

b

3D reconstruction of isolated nuclei
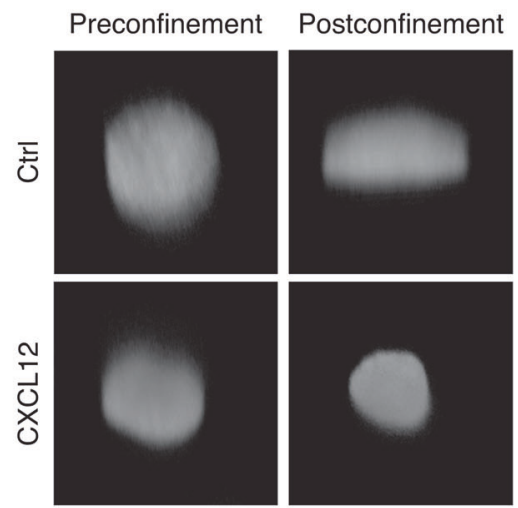

C

Jurkat cells

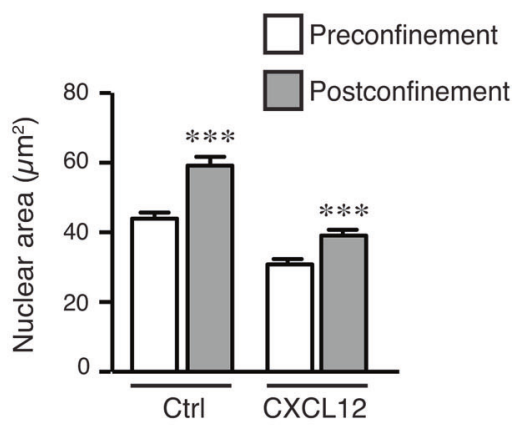

d

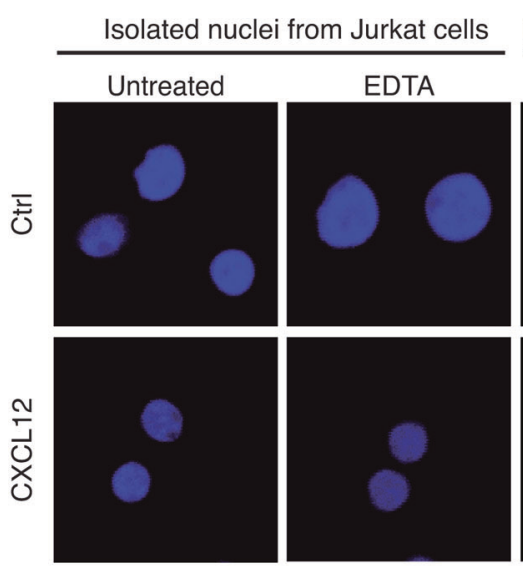
Isolated nuclei from CCRF-CEM cells Isolated nuclei from Reh cells
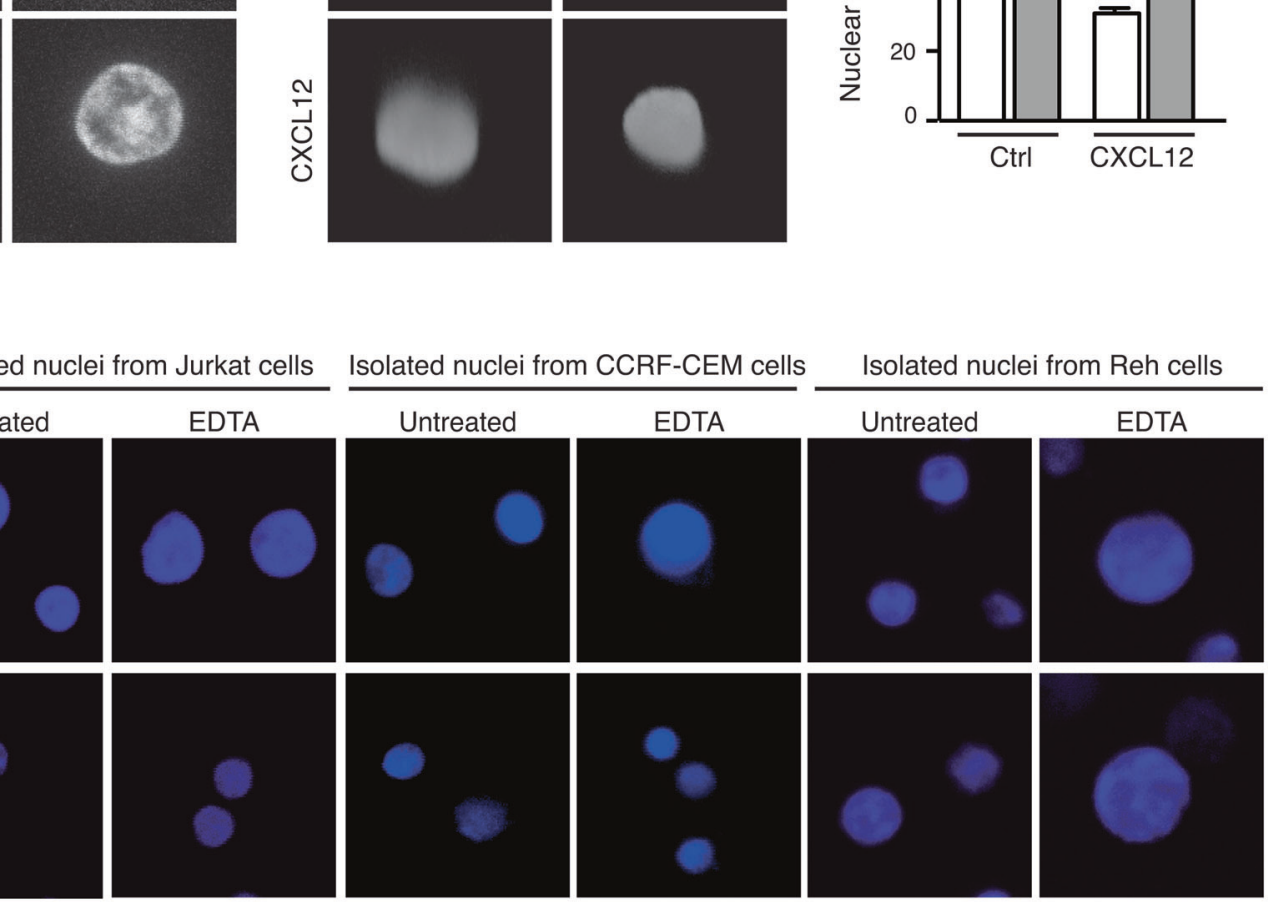

e
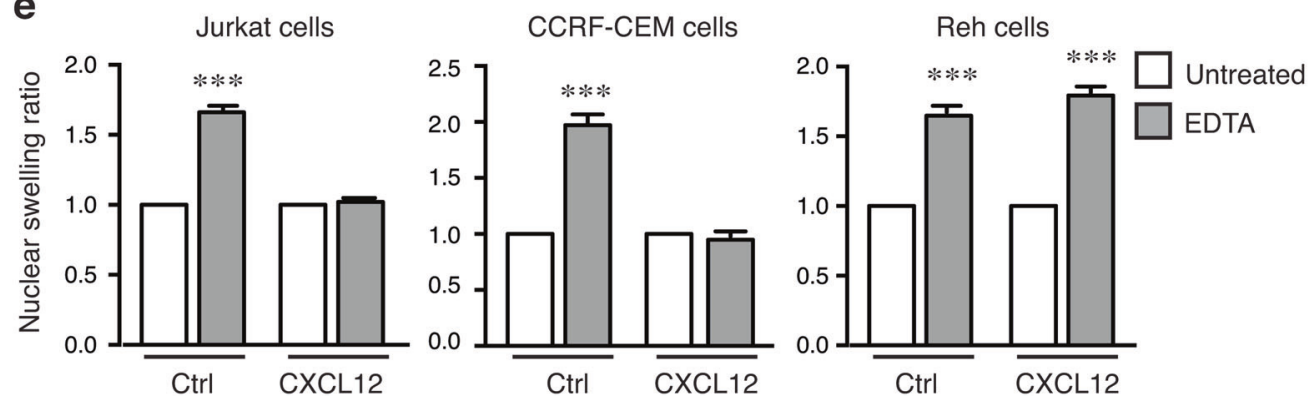

Fig. 4 Fast CXCL12 stimulation promotes mechanical changes in the nucleus of T-ALL cells. a Representative images from nuclei of Jurkat cells cultured in the absence (Ctrl) or presence of CXCL12 for $15 \mathrm{~min}$. Nuclei were isolated, stained and seeded on poly-lysine-coated coverslips. Subsequently, confocal images were acquired before and after confinement conditions ( $3 \mu \mathrm{m}$ height). b Reconstruction of 3D surface rendering of representative isolated nuclei in (a). c Graph shows the quantification of nuclear projected areas from isolated nuclei in pre- and post-constrained conditions. Mean $n=50$ nuclei \pm SEM. d Jurkat, CCRF-CEM, and Reh cells were cultured without (Ctrl) or with CXCL12 (100 ng/ml) for $15 \mathrm{~min}$. Then, nuclei were isolated and treated with EDTA to induce nuclear swelling. e Graph shows the quantification of nuclear area of isolated nuclei. Mean $n=80$ nuclei \pm SEM. $P$-values are indicated by asterisks ${ }^{* * *} P<0.001$.

cytosol of ALL cells, whilst a significant amount of the total endogenous PKC $\beta$ partitioned to the nuclear fraction of ALL cells (Supplementary Fig. S5e, f). Mechanistically, we checked whether CXCL12 stimulation might promote nuclear PKC accumulation, although we found no differences in the nuclear translocation of conventional PKC isoforms upon CXCL12 stimulation in Jurkat cells (Supplementary Fig. S5g). We pretreated T-ALL cells with PKC inhibitors and confirmed that blocking conventional PKC activity reduced Jurkat and CCRF-CEM migration in response to CXCL12 (Fig. 5f). Together, our data suggested that CXCR4 was the key receptor for the H3K9 methylation induced by $\mathrm{CXCL} 12$ and that conventional PKC activity was necessary for this nuclear change in T-ALL cells.

\section{H3K9 methyltransferases control epigenetic changes induced by CXCL12 in T-ALL cells}

Several H3K9 (histone H3 at the residue lysine K9) methyltransferases, such as suv39h1 and G9a, have been proposed as therapeutic targets against cancer cell progression and migration, including hematological malignancies $[25,26]$. To identify how 


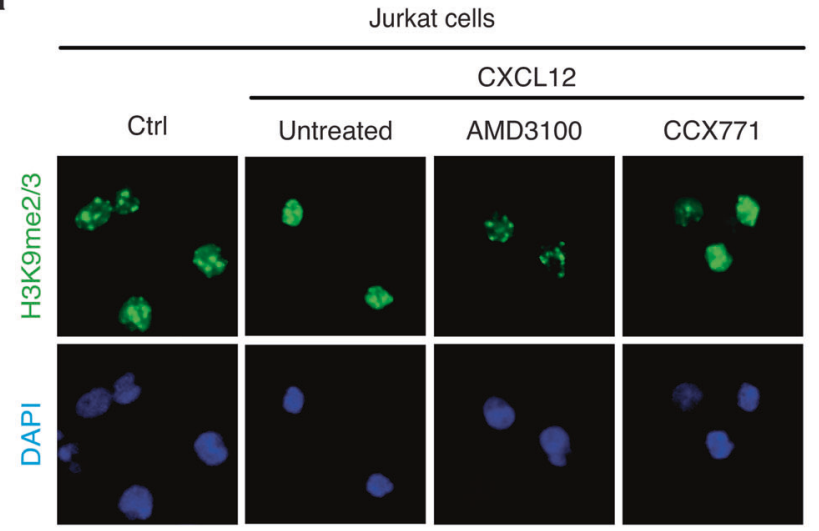

b

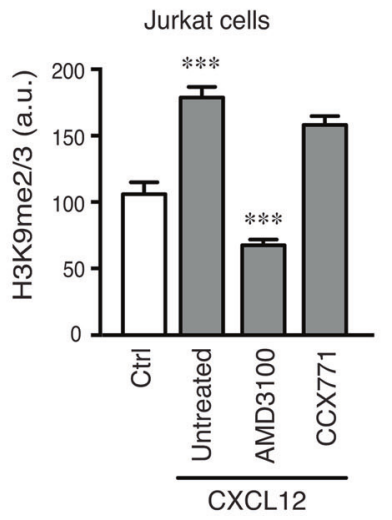

C

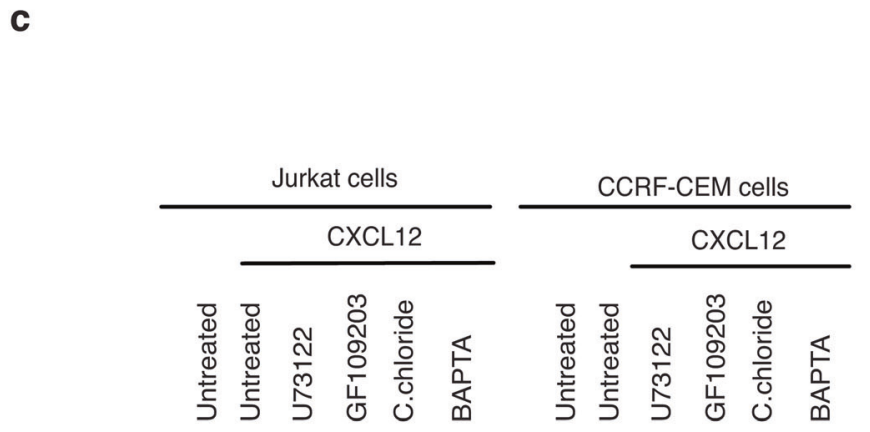

H3K9me2/3

$\mathrm{H} 3$ total

$\beta$-actin
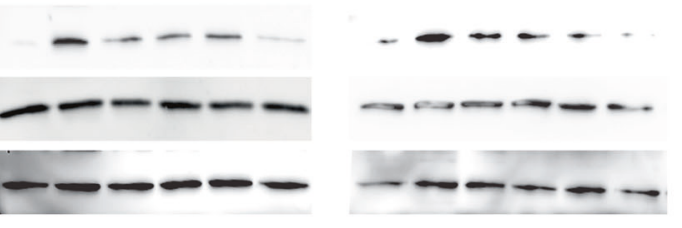

d e

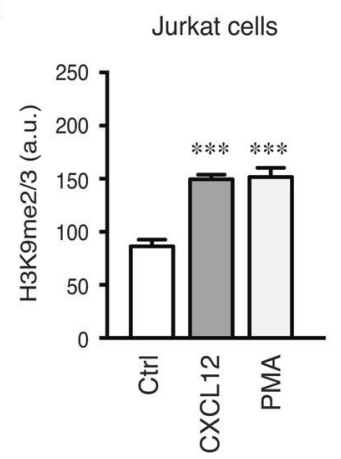

CCRF-CEM cells

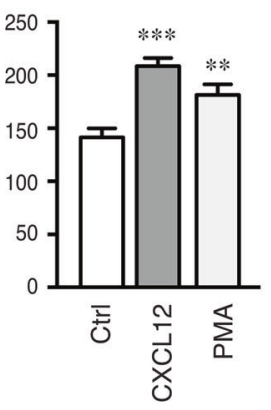

f

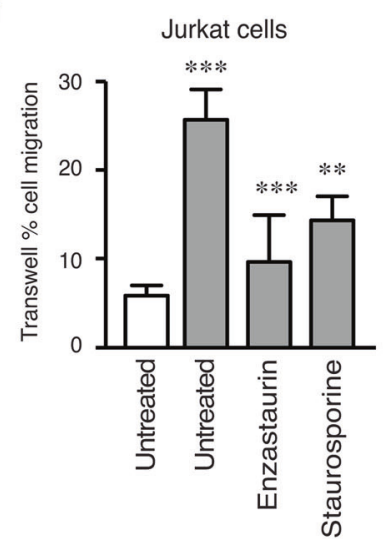

Ctrl

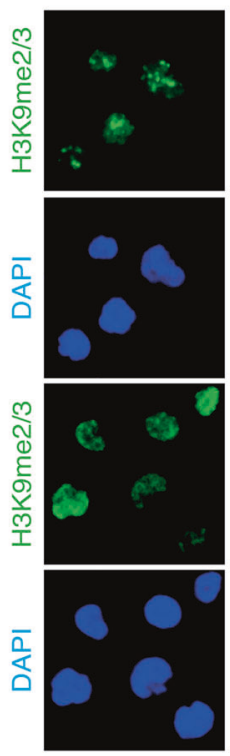

CXCL12

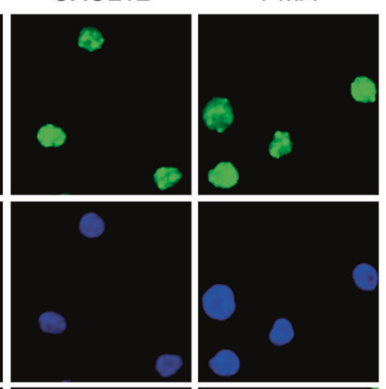

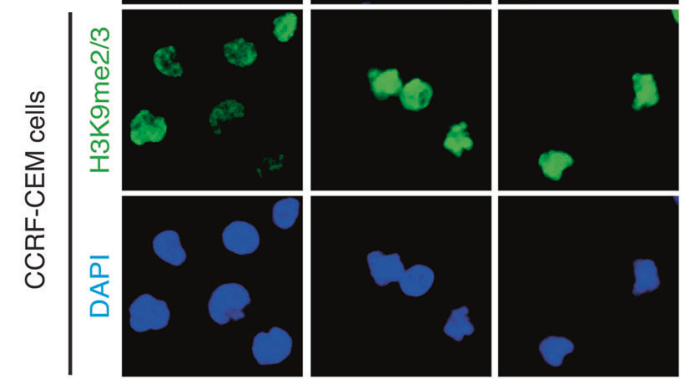

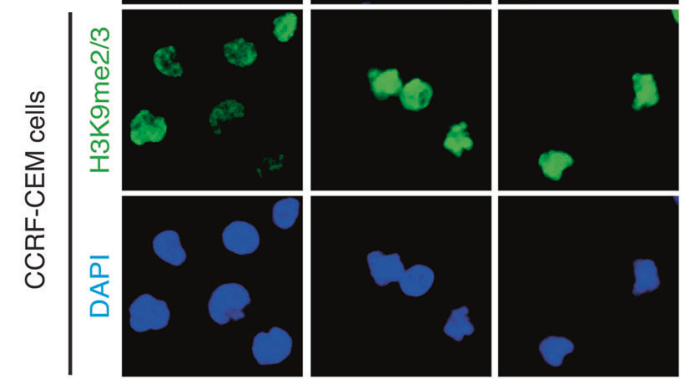

CCRF-CEM cells

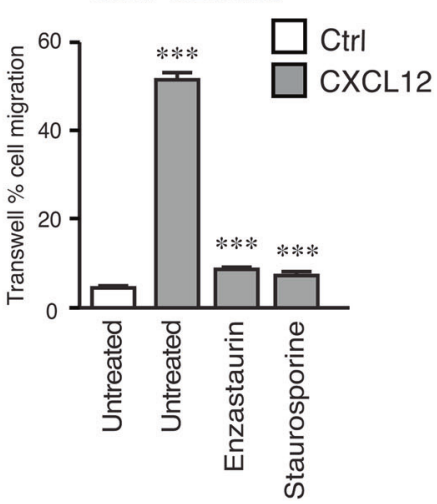

Fig. 5 Conventional PKC regulates CXCL12-medited H3K9 methylation in T-ALL cells. a Jurkat cells were preincubated with AMD3100 $(1 \mu \mathrm{M})$, CCX771 $(1 \mu \mathrm{M})$, for $30 \mathrm{~min}$ before culturing without (Ctrl) or with CXCL12. Then, cells were fixed, permeabilized and stained for the indicated markers. Confocal images show representative images of H3K9 methylation induced by CXCL12 in Jurkat and CCRF-CEM cells. b Graph shows the mean intensity (arbitrary units, a.u.) of H3K9me2/3 of ALL cell lines in (a). Mean $n=60$ cells \pm SEM. c Jurkat and CCRF-CEM cells were preincubated with U73122 $(5 \mu \mathrm{M})$, GF109203 $(2 \mu \mathrm{M})$, chelerythrine chloride $(5 \mu \mathrm{M})$ and BAPTA $(1 \mu \mathrm{M})$ for 30 min before culturing without (Ctrl) or with CXCL12. Cells were lysed, and H3K9me2/3 levels analyzed by western blotting. $\mathbf{d}$ Jurkat and CCRF-CEM cells were seeded on poly-lysine coated coverslips and stimulated with PMA $(50 \mathrm{ng} / \mathrm{ml})$ or CXCL12 $(100 \mathrm{ng} / \mathrm{ml})$ for 15 min. Then, cells were fixed, permeabilized and stained for the indicated markers. e Graph shows the mean intensity (arbitrary units, a.u.) of H3K9me2/3 of ALL cell lines in (d). Mean $n=$ 60 cells \pm SEM. $f$ Jurkat and CCRF-CEM cells were pretreated with the PKC (staurosporine, $2 \mathrm{nM}$ ) and PKC $\beta$ (enzastaurin, $50 \mathrm{nM}$ ) inhibitors for $30 \mathrm{~min}$. After $24 \mathrm{~h}$ cells in the bottom chamber were collected and counted to calculate the migration index. Mean $n=3 \pm \mathrm{SEM}$. $P$-values are indicated by asterisks $P$-values are indicated by asterisks ${ }^{* *} P<0.01,{ }^{* * *} P<0.001$. 
a

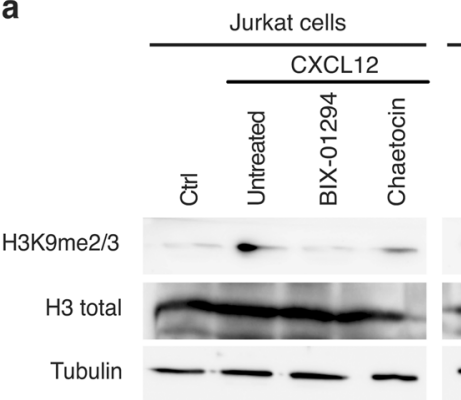

$\frac{\text { CCRF-CEM cells }}{\text { CXCL12 }}$

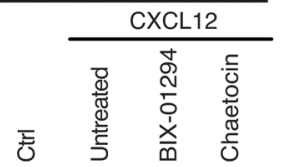

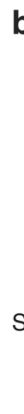

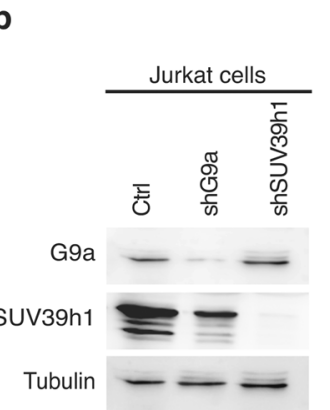

C

\begin{tabular}{l} 
Jurkat cells \\
${ }$ shG9a $}$ \\
\hline
\end{tabular}

d

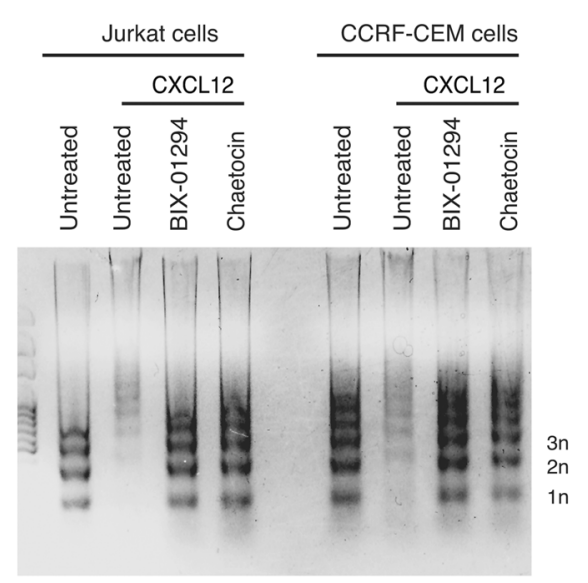

e
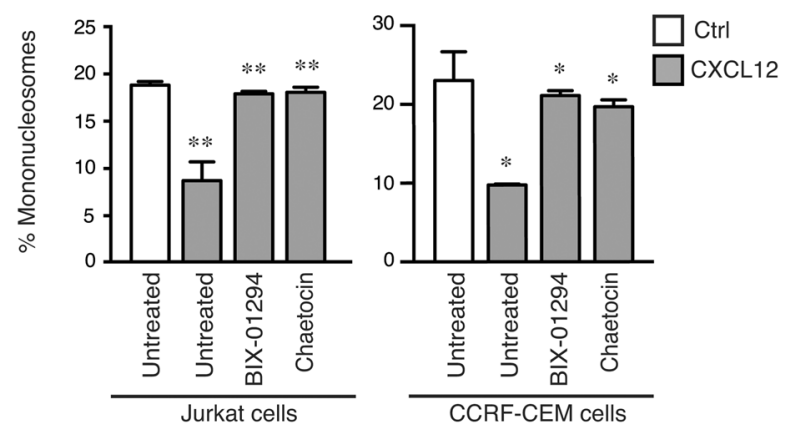

Fig. 6 The H3K9 methyltransferases G9a and suv39h1 regulate the H3K9 methylation induced by CXCL12 in T-ALL cells. a Jurkat and CCRF-CEM cells were preincubated or not with the H3K9 methyltransferase inhibitors, chaetocin (suv39h1 inhibitor $1 \mu \mathrm{M}$ ) and BIX-01294 (G9a inhibitor, $1 \mu \mathrm{M}$ ). After $30 \mathrm{~min}$, cells were cultured in the absence (Ctrl) or presence of CXCL12 (100 ng/ml) for $15 \mathrm{~min}$. Then, levels of H3K9me2/ 3 were resolved by western blotting. b Control, suv39h1- and G9a-depleted Jurkat cells were lysed and protein silencing was resolved by western blotting. c Control, suv39h1-and G9a-depleted Jurkat cells were cultured in the absence (Ctrl) or presence of CXCL12 (100 ng/ml) and their lysates analyzed by western blotting. $\mathbf{d}$ Jurkat and CCRF-CEM cells were preincubated or not with the H3K9 methyltransferase inhibitors, chaetocin $(1 \mu \mathrm{M})$ and BIX-01294 $(1 \mu \mathrm{M})$. After $30 \mathrm{~min}$, cells were cultured with CXCL12 (100 ng/ml) for 15 min, lysed, and chromatin was digested by microccocal DNAse (MNase) for $15 \mathrm{~min}$. Nucleosome releasing was resolved on $2 \%$ agarose gels. Lines indicate mono- (1n), di- (2n) and tri- $(3 \mathrm{~N})$ nucleosomes upon MNAse digestion. e Graph shows the percentage of mononucleosomes $(1 \mathrm{n})$ from $(\mathbf{d})$. ${ }^{*} P<0.05 ;{ }^{* *} P<0.01$.

H3K9 methyltransferases might catalyze the epigenetic change induced by CXCL12, we used specific inhibitors to block suv39h1 (chaetocin) and G9a (BIX-01294) activities and found that both treatments reduced significantly the $\mathrm{H} 3 \mathrm{~K} 9$ methylation induced by CXCL12 in T-ALL cells (Fig. 6a). We next examined stable Jurkat cells depleted for G9a and Suv39h1 (Fig. 6b), and confirmed that the knocking-down of these proteins abrogated the H3K9 methylation induced by CXCL12 (Fig. 6c). Given that CXCL12 stimulation promoted chromatin compaction, we investigated whether suv39h1 and G9a activities might be necessary for the protective role of $\mathrm{CXCL} 12$ to MNAse digestion. We pretreated T-ALL cells with chaetocin and BIX-01294 and observed that both inhibitors increased the sensitivity of CXCL12-stimulated cells to DNA digestion (Fig. 6d, e), confirming that CXCL12 induced H3K9 methylation was dependent on suv39h1 and G9a activities. Together, these results strongly indicate the physiological relevance of H3K9 HMTs to control epigenetic changes and chromatin compaction induced by CXCL12 in T-ALL cells.

\section{Targeting H3K9 methylation reduces the invasiveness of ALL cells in vitro and in vivo}

Nuclear constrictions are related to cell migration and promote the redistribution of multiple nuclear components, including histone markers [27]. We then sought to quantify the distribution of H3K9 methylation in migrating ALL cells by determining the nuclear signal of $\mathrm{H} 3 \mathrm{~K} 9 \mathrm{me} 2 / 3$ and the nuclear contour. We found that deformable migrating cells showed higher H3K9me2/3 intensity in those areas corresponding to active nuclear squeezing through $3 \mu \mathrm{m}$ Transwell pores in response to $\mathrm{CXCL12}$ gradient (Supplementary Movie S1, Fig. 7a, b). Furthermore, constricted migration of CCRF-CEM and Reh cells also promoted the polarization of $\mathrm{H} 3 \mathrm{~K} 9 \mathrm{me} 2 / 3$ signal at the nuclear front of migrating cells (Supplementary Fig. S6a). To address how the nuclear changes induced by CXCL12 might impact the ALL cell capacity to migrate through small pores, we analyzed the chemotactic response of T- and B-ALL cells through $3 \mu \mathrm{m}$ pores and found that Jurkat and CCRF-CEM cells increased their migration index (2 and 1.6 times, respectively) in response to CXCL12 for $3 \mathrm{~h}$, whilst Reh cells did not alter their migration index (Supplementary Fig. $\mathrm{S} 6 \mathrm{~b})$. We analyzed the chemotactic response at longer times (24 h) of primary cells from patients with ALL and confirmed that T-ALL cells increased their migration (Fig. 7c). Based on these findings, we quantified the cell migration of T-ALL cells upon blocking or silencing $\mathrm{H} 3 \mathrm{~K} 9$ methyltransferases. We found that targeting $\mathrm{H} 3 \mathrm{~K} 9$ methylation impaired Jurkat cell migration in response to CXCL12 (Fig. 7d, e). Similarly, we confirmed that chaetocin or BIX-01294 treatments reduced the migration of CCRF-CEM cells induced by CXCL12 (Supplementary Fig. S6c), confirming that H3K9 methylation activity might play a fundamental role in CXCL12-mediated migration of T-ALL cells through constricted conditions.

Constricted microchannels have been developed to mimic rigid environments that require the active deformability of migrating cells [28]. We observed that pretreatment with H3K9 methyltransferase inhibitors reduced the total number of Jurkat cells coming into the microchannels (Fig. 8a, Supplementary Movies S2-S4). To further elucidate whether H3K9 methylation might regulate nuclear 
a

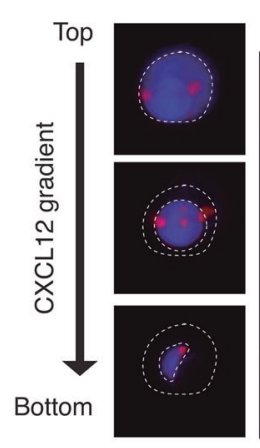

C

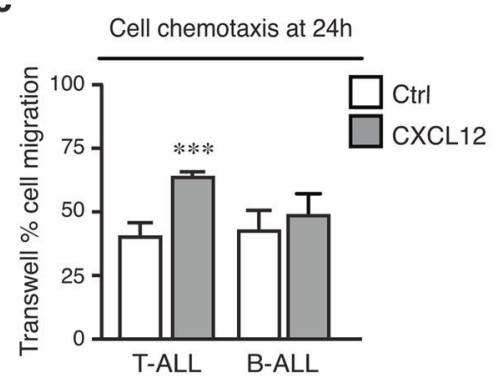

d

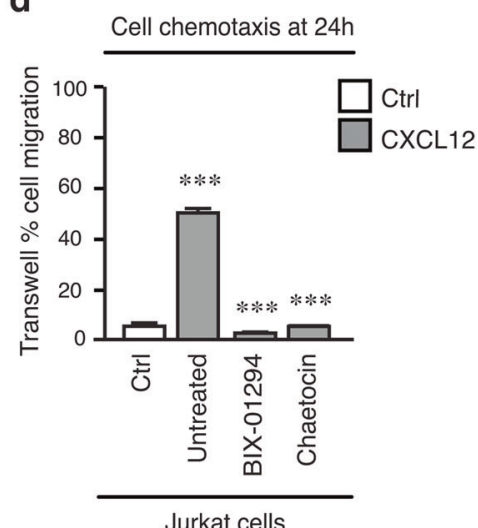

b

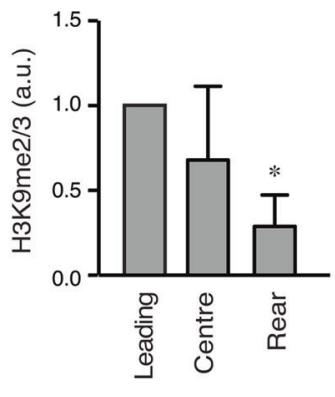

e

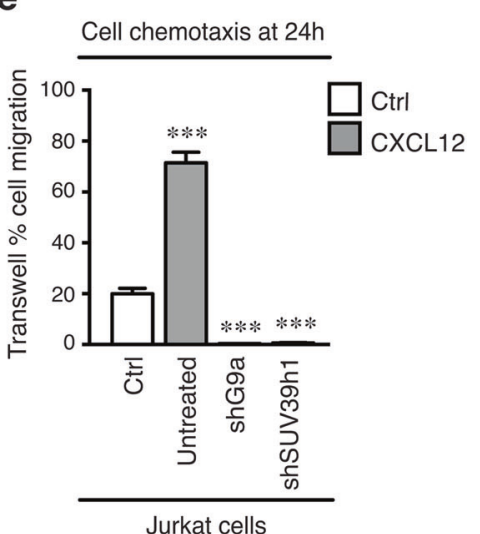

Fig. 7 H3K9 methylation controls T-ALL chemotaxis through rigid pores in response to CXCL12. a Representative image of a Jurkat cell migrating through a $3 \mu \mathrm{m}$ pore in response to CXCL12 gradient. Cells were fixed, permeabilized and stained for the indicated proteins. White lines indicate the nuclear area and the nuclear constriction through the small pore. Right panel shows a 3D reconstruction of migrating cell nucleus squeezing, and white arrows indicates $\mathrm{H} 3 \mathrm{~K} 9 \mathrm{me} 3$ signal accumulating at the nuclear constriction. $\mathbf{b}$ Graph shows the H3K9me2/3 ratio normalized to DAPI at the leading area of the nucleus (highly deformable region). H3K9 methylation accumulates mainly at the nuclear front during cell deformation across the small pore. c Chemotaxic migration of primary T- and B-ALL cells through $3 \mu \mathrm{m}$ pore transwells towards CXCL12 $(100 \mathrm{ng} / \mathrm{ml})$ gradient. After $24 \mathrm{~h}$, cells were collected from the bottom chamber and counted to calculate the migration index. Mean $n$ $=3 \pm$ SEM. d Jurkat cells were pretreated or not with chaetocin $(1 \mu \mathrm{M})$ and BIX-01294 $(1 \mu \mathrm{M})$ for $1 \mathrm{~h}$ prior to adding them in the upper chamber of the transwell. Then, cells were allowed to migrate through $3 \mu \mathrm{m}$ pore transwells toward CXCL12 $(100 \mathrm{ng} / \mathrm{ml}) \mathrm{gradient}$. After $24 \mathrm{~h}$, cells were collected from the bottom chamber and counted to calculate the migration index. Mean $n=3 \pm$ SEM. e Control, suv39h1- and G9a-depleted Jurkat cells were seeded in the upper chamber of the transwell and CXCL12 $(100 \mathrm{ng} / \mathrm{ml})$ was added to the bottom chamber. After $24 \mathrm{~h}$, cells were collected from the bottom chamber and counted to calculate the migration index. Mean $n=3 \pm$ SEM. $P$-values are indicated by asterisks ${ }^{*} P<0.05 ;{ }^{* * *} P<0.001$.

deformability and cell migration across confined spaces, we investigated the percentage of migrating cells that penetrate and deform effectively across constrictions. We observed that cell invasiveness (defined as cells migrating through constrictions) was reduced by chaetocin or BIX-01294 treatments (Fig. 8b). Together, our results suggested that $\mathrm{H} 3 \mathrm{~K} 9$ methylation played a fundamental role in CXCL12-mediated migration and deformability of T-ALL cells. To evaluate the therapeutic potential of H3K9 methyltransferase inhibitors against leukemia dissemination, we mixed control and pretreated Jurkat cells with H3K9 methyltransferase inhibitors, ad injected them into immunodeficient recipient mice. At $3 \mathrm{~h}$ after transplantation, we compared the presence of control and pretreated cells into the BM and spleen by flow cytometry (Fig. 8c). We observed that H3K9 methyltransferase inhibition impaired the homing of Jurkat cells into the spleen and BM compared to untreated cells (Fig. $8 \mathrm{~d}$, e). Finally, to confirm the consequences of the in vivo effects of H3K9 methyltransferase inhibitors in T-ALL cell migration, we pretreated CCRF-CEM cells with BIX-01294 and chaetocin, and analyzed the homing of leukemic cells into the spleen and BM at $3 \mathrm{~h}$ after inoculation (Supplementary Fig. S7a). We demonstrated that both inhibitors also reduced significantly the number of CCRF-CEM cells that reached the BM and spleen (Supplementary Fig. S7b, c).
Together, these data suggested that $\mathrm{H} 3 \mathrm{~K} 9$ methylation plays a role in cell squeezing and migration of T-ALL cells.

\section{DISCUSSION}

T-ALL cells have to penetrate physical barriers to infiltrate various organs (including lymph nodes, liver, spleen, BM, and central nervous system) [29]. These places serve as protected niches against conventional chemotherapies and facilitate ALL recurrence and relapse $[30,31]$. Here, we demonstrated that CXCL12 determines the H3K9 methylation, the biomechanical response and the invasiveness of T-ALL cells in vitro and in vivo.

Several chemokines mediate the infiltration and progression of ALL pathology [32]. CXCL12 produced by resident cells at tumor niches plays a critical role in the homing, proliferation, and survival of ALL cells into the BM, liver, CNS, etc. [22, 33, 34]. We observed that CXCL12 promoted rapid epigenetic changes within minutes in leukemia cells. Strikingly, only T-ALL cells upregulated H3K9me2/3 levels, while B-ALL cells showed diminished levels of H3K9 methylation upon CXCL12 stimulation. Differences in the epigenetic stage of B- or T-ALL cells have been previously pointed, including the hypermethylation of Notch factors and that only 
a

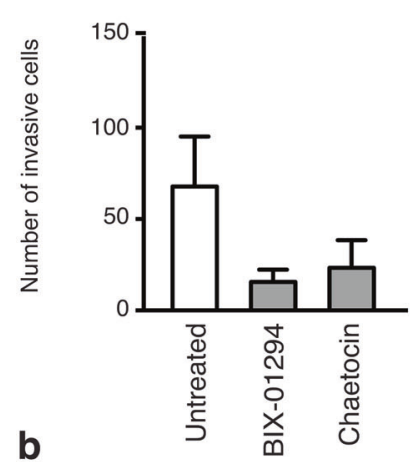

b

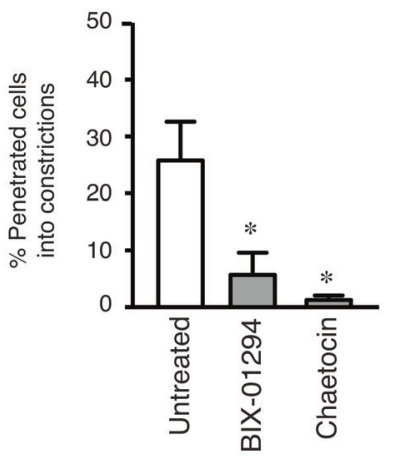

d

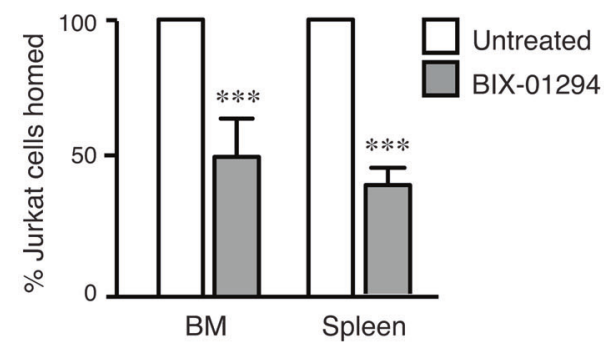

C
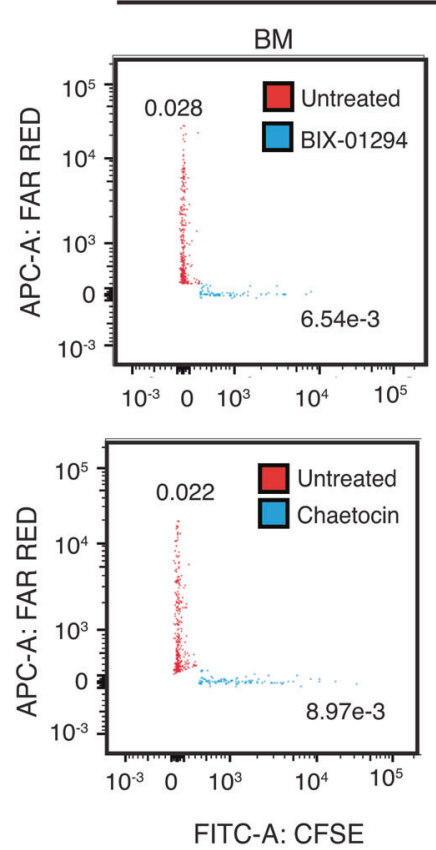

Jurkat cells

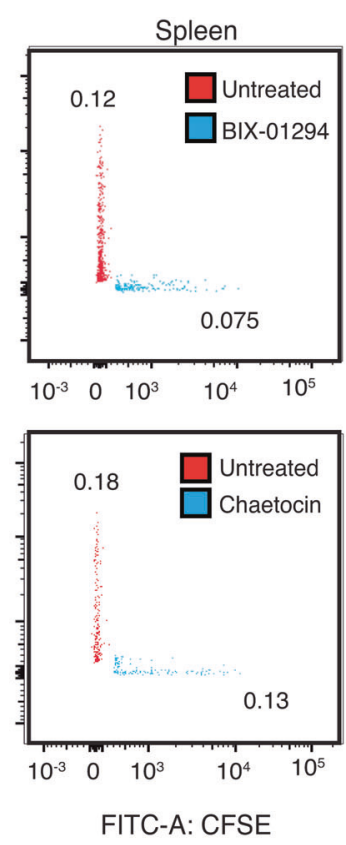

e

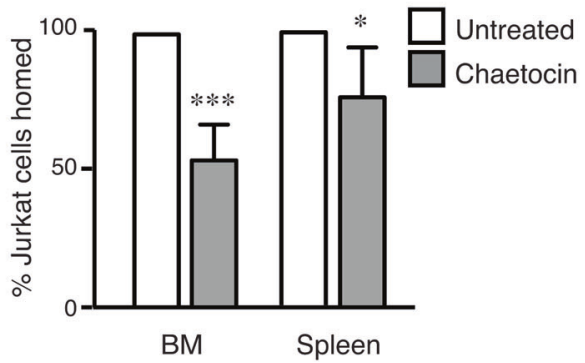

Fig. 8 H3K9 methylation regulates nuclear constrictions and T-ALL migration in vitro and in vivo systems. a Jurkat cells were pretreated or not with chaetocin $(1 \mu \mathrm{M})$ and BIX-01294 $(1 \mu \mathrm{M})$ for $1 \mathrm{~h}$ prior adding them into the microchannel chamber. Cells were allowed to enter in the microchannel upon CXCL12 stimulation for $8 \mathrm{~h}$. Graph represents the total number of cells migrating into the microchannels Mean $n=3 \pm$ SEM. b Graph shows the percentage of cells that effectively entered in the microchannel and crossed through constrictions. Mean $n=3 \pm$ SEM. c Jurkat cells were labeled with $5 \mu$ M CFSE (for BIX-01294- or chaetocin-pretreated cells) and $1 \mu$ M Far Red Cell Tracker (untreated cells). Then, BIX-01294- or chaetocin-pretreated cells were mixed (1:1) with untreated cells, and the mixture was injected into the tail vein of NSG mice $\left(5 \times 10^{6}\right.$ cells/mouse). Five mice were used for each combination and a negative control mouse received only RPMI. After $3 \mathrm{~h}$, both populations of labeled cells in the spleen and bone marrow were determined by flow cytometry. Numbers indicate the percentage of cells per sample analyzed. d Graphs show the percentage of untreated or BIX-01294-pretreated cells that homed into the bone marrow or spleen. Numbers were normalized according to the total number of cells $/ \mathrm{ml}$ in that organ and the percentage of green or red cells and the number of cells $/ \mathrm{ml}$ injected. e Graphs show the percentage of untreated or chaetocin-pretreated cells that homed into the bone marrow or spleen. $P$-values are indicated by asterisks $\left({ }^{*} P<0.05 ;{ }^{*} P<0.01 ;{ }^{* *} P<0.001\right)$.

B-ALL (but not T-ALL) cells respond to decitabine (which promotes DNA demethylation) treatment [35]. Our results suggest that Tand B-ALL cells might use different migratory mechanisms to reach and colonize other tissues, and confirm that different epigenetic pathways may be lineage-specific. In agreement with cell proliferation changes induced by $\mathrm{CXCL12}$, we observed that short times of CXCL12 stimulation might promote T- and B-ALL cell proliferation, although H3K9 methylation induced by CXCL12 might occur at shorter times and in response to other biomechanical or migratory stimuli.

ALL cells express several chemokine receptors, including CXCR4, which is associated with worse outcomes in pediatric patients [36]. Furthermore, it has been reported that CXCR4 promotes tumorigenicity and transcriptional changes in multiple cell types
[37]. There is a correlation between ALL infiltration in vivo and the levels of CXCR4, CCR7, and ZAP70 (Zeta-chain-associated protein kinase 70) [38]. Our data provide evidence for CXCR4 as a major mediator in the H3K9 methylation induced by CXCL12. Although CXCR7 influences the signaling induced by CXCR4 [39], we did not find any significant effect blocking CXCR7 on the CXCL12mediated H3K9 methylation. In addition, a recent study showed that the CXCR7/CXCR4 heterodimer regulates the histone demethylase JMJD2D and induces H3K9 and H3K36 demethylation [40], which aligns with our observations that the upregulation of H3K9 methylation in T-ALL cells is independent of CXCR7 signaling.

Mechanistically, PKC is a molecular pathway involved in the CXCL12 signaling of immune cells [41]. Several PKC isoforms, 
including $P K C \zeta, P K C a$, and $P K C \beta$, influence the chemotaxis and in vivo engraftment of CD34 + cells towards CXCL12 [42]. It has been reported that PMA and CXCL12 induce different migration and morphology in normal T-cells [43]. Here, we described that PMA stimulation promoted similar H3K9 methylation in $\mathrm{T}-$ and B-ALL cells, whilst CXCL12-stimulated H3K9 methylation only in T-ALL cells. This suggests that PKC activation might be sufficient to induce this epigenetic change in both cell types, although only T-ALL cells might activate this pathway in response to CXCL12. The BCR controls the CXCR4 internalization and signaling in B-cells that might explain the different responsiveness observed to CXCL12 stimulation in B-ALL cells [44]. Also, it has been reported that cortactin is important for CXCR4 internalization upon CXCL12 stimulation in T- but not in B-ALL cells [45]. Our findings also indicated that CXCL12-mediated H3K9 methylation was dependent on conventional PKC activity. Interestingly, CXCR7 does not regulate $\mathrm{Ca} 2$ mobilization [39]. This evidence could explain why conventional PKC activity and CXCR4, but not CXCR7, would be necessary to induce H3K9 methylation in T-ALL cells. It has been reported that most of these isoforms are in the cytosol of T-lymphocytes [43], we observed that PKC $\beta$ might localize in the nucleus of T-ALL cells, whilst PKCa showed more cytoplasmic localization. This aligned with previous reports showing the nuclear localization of PKC isoforms and their role on gene expression, immune cell differentiation and phosphorylating nuclear components, such as transcription factors, lamins and histones $[46,47]$. PKC activity may therefore be a key element of H3K9 methylation machinery.

So far, cancer cells present altered histone modifications and other epigenetic changes, which correlate with aberrant genomic expression features according to the chromatin structure [48]. H3K9 methylation is widely involved in gene silencing and transcriptional repression [16]. The study of the transcriptome and methylome is critical to identify the progression of ALL in pediatric patients [49]. It has been reported that CXCL12 regulates LIC (leukemia initiating cell) biology in protective niches to resist conventional therapies $[50,51]$. By using ChIP-seq, we observed that fast stimulation of ALL cells with CXCL12 induced an increment in the number of $\mathrm{H} 3 \mathrm{~K} 9 \mathrm{me} 3$ peaks without significant correlation at a transcriptional level. This discrepancy between gene transcription and H3K9 methylation variance has been previously reported [52] and could reflect a direct effect of the chromatin on the nuclear mechanics rather than its transcriptional role at longer times or during leukemia maintenance and progression.

Physical barriers induce nuclear and chromatin changes in cancer cells [53], and increasing evidence demonstrates that the chromatin contributes to the mechanical response of the nucleus [20]. Interestingly, we observed that both T- and B-ALL cells also redistributed $\mathrm{H} 3 \mathrm{~K} 9 \mathrm{me} 3$ marker at the nuclear front of migrating cells, even when B-ALL cells did not upregulate $\mathrm{H} 3 \mathrm{~K} 9$ methylation in response to CXCL12. This suggests that CXCL12 affected the chromatin compaction of T-ALL cells and, on the other hand, the chromatin distribution during constricted cell migration might occur independently of the leukemia cell type. This agrees with the idea of constricted migration causes DNA damage and affects the cell cycle of tumor cells [54]. In our study, we determined that CXCL12 promoted effective changes in the mechanical deformability response of nuclei from T-ALL cells. The role of $\mathrm{H} 3 \mathrm{~K} 9$ methylation in the invasiveness of multiple cancer cells has been previously suggested $[55,56]$. Furthermore, it has been reported a link between H3K9 methylation activity and transendothelial migration of ALL cells [57]. CXCL12/CXCR4 interaction is required for the chemotaxis of T-ALL cells into the BM and disease progression in mouse models. Targeting CXCR4 with inhibitors or antagonisms, such as POL5551 and plerixafor, blocks ALL engraftment in vivo and increase the efficacy of conventional treatments [58, 59]; however, conventional treatments upregulate CXCR4 in ALL cells and treatments against CXCL12 and CXCR4 may cause side effects on other cell receptors involved in cell migration and adhesion [60]. Since H3K9 methylation regulated the cell migration and nuclear deformability of T-ALL, blockage of this process may be a promising therapy for patients. Here, we found that blocking suv39h1 and G9a activities in T-ALL cells reduced their capacity to reach the $\mathrm{BM}$ and the spleen. It is noteworthy that other cell receptors might regulate leukemia cell dissemination, including integrins [61], and G9a is a promising therapeutic target in blood cancers [62] and might contribute to altering the epigenetic landscape of ALL in the migration process in response to other external stimuli. Previous studies have indicated that H3K9 methyltransferase inhibitors block the growth of tumor cells in xenograft models [63-66]. Of particular note is that these studies were performed on established tumors and drug administration for longer periods rather than defining the direct role of these treatments on direct cell invasion. Our results showed that the use of H3K9 methyltransferase inhibitors might reflect a direct impact on leukemia cell migration. This aligns with previous anti-tumor effects of these inhibitors on cancer cells, such as oxidative stress and cell death and opens potential implications in a complex metastatic situation, which includes a sequence of events related to cell adhesion, migration, survival, and tumor niche engraftment. Together, we might speculate that the use of $\mathrm{H} 3 \mathrm{~K} 9$ methyltransferase inhibitors might represent a therapeutic option to block different stages of T-ALL infiltration and progression.

\section{REFERENCES}

1. Pui $\mathrm{CH}$, Robison LL, Look AT. Acute lymphoblastic leukaemia. Lancet. 2008;371:1030-43.

2. Aifantis I, Raetz E, Buonamici S. Molecular pathogenesis of T-cell leukemia and lymphoma. Nat Rev Immunol. 2008;8:380-90.

3. Pui $\mathrm{CH}$, Jeha $\mathrm{S}$. New therapeutic strategies for the treatment of acute lymphoblastic leukaemia. Nat Rev Drug Disco. 2007;6:149-65.

4. Moser B, Loetscher P. Lymphocyte traffic control by chemokines. Nat Immunol. 2001;2:123-128.

5. Sugiyama T, Kohara $H$, Noda $M$, Nagasawa T. Maintenance of the hematopoietic stem cell pool by CXCL12-CXCR4 chemokine signaling in bone marrow stromal cell niches. Immunity. 2006;25:977-88.

6. de Lourdes Perim A, Amarante MK, Guembarovski RL, de Oliveira CE, Watanabe MA. CXCL12/CXCR4 axis in the pathogenesis of acute lymphoblastic leukemia (ALL): a possible therapeutic target. Cell Mol Life Sci. 2015;72:1715-23.

7. Pitt LA, Tikhonova AN, Hu H, Trimarchi T, King B, Gong Y, et al. CXCL12-producing vascular endothelial niches control acute $\mathrm{T}$ cell leukemia maintenance. Cancer Cell. 2015;27:755-68.

8. Scupoli MT, Donadelli M, Cioffi F, Rossi M, Perbellini O, Malpeli G, et al. Bone marrow stromal cells and the upregulation of interleukin-8 production in human T-cell acute lymphoblastic leukemia through the CXCL12/CXCR4 axis and the NFkappaB and JNK/AP-1 pathways. Haematologica. 2008;93:524-32.

9. Cancilla D, Rettig MP, DiPersio JF. Targeting CXCR4 in AML and ALL. Front Oncol. 2020;10:1672.

10. Hong Z, Wei Z, Xie T, Fu L, Sun J, Zhou F, et al. Targeting chemokines for acute lymphoblastic leukemia therapy. J Hematol Oncol. 2021;14:48.

11. Rea S, Eisenhaber F, O'Carroll D, Strahl BD, Sun ZW, Schmid M. et al. Regulation of chromatin structure by site-specific histone H3 methyltransferases. Nature. 2000;406:593-9.

12. Friedl $P$, Wolf $K$, Lammerding J. Nuclear mechanics during cell migration. Curr Opin Cell Biol. 2011;23:55-64.

13. Pfeifer CR, Irianto J, Discher DE. Nuclear mechanics and cancer cell migration. Adv Exp Med Biol. 2019;1146:117-30.

14. Stephens AD, Banigan EJ, Marko JF. Chromatin's physical properties shape the nucleus and its functions. Curr Opin Cell Biol. 2019;58:76-84.

15. Schumann K, Lammermann T, Bruckner M, Legler DF, Polleux J, Spatz JP, et al. Immobilized chemokine fields and soluble chemokine gradients cooperatively shape migration patterns of dendritic cells. Immunity. 2010;32:703-13.

16. Nakayama J, Rice JC, Strahl BD, Allis CD, Grewal SI. Role of histone H3 lysine 9 methylation in epigenetic control of heterochromatin assembly. Science. 2001;292:110-3. 
17. Kidiyoor GR, Li Q, Bastianello G, Bruhn C, Giovannetti I, Mohamood A, et al. ATR is essential for preservation of cell mechanics and nuclear integrity during interstitial migration. Nat Commun. 2020;11:4828.

18. Bendall LJ, Baraz R, Juarez J, Shen W, Bradstock KF. Defective p38 mitogenactivated protein kinase signaling impairs chemotaxic but not proliferative responses to stromal-derived factor-1alpha in acute lymphoblastic leukemia. Cancer Res. 2005;65:3290-8.

19. Pajerowski JD, Dahl KN, Zhong FL, Sammak PJ, Discher DE. Physical plasticity of the nucleus in stem cell differentiation. Proc Natl Acad Sci USA. 2007;104:15619-24.

20. Stephens AD, Banigan EJ, Adam SA, Goldman RD, Marko JF. Chromatin and lamin A determine two different mechanical response regimes of the cell nucleus. Mol Biol Cell. 2017;28:1984-96.

21. Le Berre M, Aubertin J, Piel M. Fine control of nuclear confinement identifies a threshold deformation leading to lamina rupture and induction of specific genes. Integr Biol (Camb). 2012;4:1406-14.

22. Jost TR, Borga C, Radaelli E, Romagnani A, Perruzza L, Omodho L, et al. Role of CXCR4-mediated bone marrow colonization in CNS infiltration by $\mathrm{T}$ cell acute lymphoblastic leukemia. J Leukoc Biol. 2016;99:1077-87.

23. Melo RCC, Longhini AL, Bigarella CL, Baratti MO, Traina F, Favaro P. et al. CXCR7 is highly expressed in acute lymphoblastic leukemia and potentiates CXCR4 response to CXCL12. PLoS One. 2014;9:e85926

24. Wang JF, Park IW, Groopman JE. Stromal cell-derived factor-1alpha stimulates tyrosine phosphorylation of multiple focal adhesion proteins and induces migration of hematopoietic progenitor cells: roles of phosphoinositide-3 kinase and protein kinase C. Blood. 2000;95:2505-13.

25. Lai YS, Chen JY, Tsai HJ, Chen TY, Hung WC. The SUV39H1 inhibitor chaetocin induces differentiation and shows synergistic cytotoxicity with other epigenetic drugs in acute myeloid leukemia cells. Blood. Cancer J. 2015;5:e313.

26. Lu C, Klement JD, Yang D, Albers T, Lebedyeva IO, Waller JL. et al. SUV39H1 regulates human colon carcinoma apoptosis and cell cycle to promote tumor growth. Cancer Lett. 2020;476:87-96.

27. Irianto J, Xia Y, Pfeifer CR, Greenberg RA, Discher DE. As a nucleus enters a small pore, chromatin stretches and maintains integrity, even with DNA breaks. Biophys J. 2017;112:446-9.

28. Vargas $P$, Terriac E, Lennon-Dumenil AM, Piel M. Study of cell migration in microfabricated channels. J Vis Exp. 2014;84:e51099.

29. Cannon JL, Oruganti SR, Vidrine DW. Molecular regulation of T-ALL cell infiltration into the CNS. Oncotarget. 2017;8:84626-7.

30. Frishman-Levy L, Izraeli S. Advances in understanding the pathogenesis of CNS acute lymphoblastic leukemia and potential for therapy. $\mathrm{Br} J$ Haematol. 2017;176:157-67.

31. Vadillo $E$, Dorantes-Acosta $E$, Pelayo $R$, Schnoor M. T cell acute lymphoblastic leukemia (T-ALL): New insights into the cellular origins and infiltration mechanisms common and unique among hematologic malignancies. Blood Rev. 2018;32:36-51.

32. Gomez AM, Martinez C, Gonzalez M, Luque A, Melen GJ, Martínez J, et al. Chemokines and relapses in childhood acute lymphoblastic leukemia: $\mathrm{A}$ role in migration and in resistance to antileukemic drugs. Blood Cells Mol Dis. 2015;55:220-7.

33. Bradstock KF, Makrynikola V, Bianchi A, Shen W, Hewson J, Gottlieb DJ. Effects of the chemokine stromal cell-derived factor- 1 on the migration and localization of precursor-B acute lymphoblastic leukemia cells within bone marrow stromal layers. Leukemia. 2000;14:882-8.

34. Kato I, Niwa A, Heike T, Fujino H, Saito MK, Umeda K, et al. Identifcation of hepatic niche harboring human acute lymphoblastic leukemic cells via the SDF-1/CXCR4 axis. PLoS ONE. 2011;6:e27042.

35. Kuang SQ, Fang Z, Zweidler-McKay PA, Yang H, Wei Y, Gonzalez-Cervantes EA, et al. Epigenetic inactivation of Notch-Hes pathway in human B-cell acute lymphoblastic leukemia. PLoS One. 2013;8:e61807.

36. Crazzolara R, Kreczy A, Mann G, Heitger A, Eibl G, Fink FM. et al. High expression of the chemokine receptor CXCR4 predicts extramedullary organ infiltration in childhood acute lymphoblastic leukaemia. Br J Haematol. 2001;115:545-53.

37. Bao Y, Wang Z, Liu B, Lu X, Xiong Y, Shi J, et al. A feed-forward loop between nuclear translocation of CXCR4 and HIF-1alpha promotes renal cell carcinoma metastasis. Oncogene. 2019;38:881-95.

38. Alsadeq A, Fedders $H$, Vokuhl C, Belau NM, Zimmermann M, Wirbelauer $T$, et al. The role of ZAP70 kinase in acute lymphoblastic leukemia infiltration into the central nervous system. Haematologica. 2017;102:346-55.

39. Levoye A, Balabanian K, Baleux F, Bachelerie F, Lagane B. CXCR7 heterodimerizes with CXCR4 and regulates CXCL12-mediated $\mathrm{G}$ protein signaling. Blood. 2009;113:6085-93.

40. Song ZY, Wang F, Cui SX, Gao ZH. Qu XJ.CXCR7/CXCR4 heterodimer-induced histone demethylation: a new mechanism of colorectal tumorigenesis. Oncogene. 2019;38:1560-75.
41. Altman A, Kong KF. Protein kinase $C$ enzymes in the hematopoietic and immune systems. Annu Rev Immunol. 2016;34:511-38.

42. Petit I, Goichberg P, Spiegel A, Peled A, Brodie C, Seger R. et al. Atypical PKC-zeta regulates SDF-1-mediated migration and development of human CD34+ progenitor cells. J Clin Invest. 2005;115:168-76.

43. Wei SY, Lin TE, Wang WL, Lee PL, Tsai MC, Chiu JJ. Protein kinase $C-\delta$ and $-\beta$ coordinate flow-induced directionality and deformation of migratory human blood T-lymphocytes. J Mol Cell Biol. 2014;6:458-72.

44. Guinamard R, Signoret N, Ishiai M, Marsh M, Kurosaki T, Ravetch JV. B cell antigen receptor engagement inhibits stromal cell-derived factor (SDF)-1alpha chemotaxis and promotes protein kinase C (PKC)-induced internalization of CXCR4. J Exp Med. 1999;189:1461-6.

45. Velázquez-Avila $M$, Balandrán JC, Ramírez-Ramírez $D$, Velázquez-Avila $M$, Sandoval A, Felipe-López A, et al. High cortactin expression in B-cell acute lymphoblastic leukemia is associated with increased transendothelial migration and bone marrow relapse. Leukemia. 2019;33:1337-48.

46. Poli A, Ratti S, Finelli C, Mongiorgi S, Clissa C, Lonetti A, et al. Nuclear translocation of PKC-alpha is associated with cell cycle arrest and erythroid differentiation in myelodysplastic syndromes (MDSs). FASEB J. 2018;32:681-92.

47. Edens LJ, Dilsaver MR, Levy DL. PKC-mediated phosphorylation of nuclear lamins at a single serine residue regulates interphase nuclear size in Xenopus and mammalian cells. Mol Biol Cell. 2017;28:1389-99.

48. Esteller M. Epigenetics in cancer. N. Engl J Med. 2008;358:1148-59.

49. Almamun M, Levinson BT, van Swaay AC, Johnson NT, McKay SD, Arthur GL, et al. Integrated methylome and transcriptome analysis reveals novel regulatory elements in pediatric acute lymphoblastic leukemia. Epigenetics. 2015;10:882-90.

50. Konopleva MY, Jordan CT. Leukemia stem cells and microenvironment: biology and therapeutic targeting. J Clin Oncol. 2011;29:591-9.

51. Passaro D, Irigoyen M, Catherinet C, Gachet $S$, Da Costa C, Lasgi C, et al. CXCR4 is required for Leukemia-Initiating Cell activity in T cell acute lymphoblastic leukemia. Cancer Cell. 2015;27:769-79.

52. Nava MM, Miroshnikova YA, Biggs LC, Whitefield DB, Metge F, Boucas J, et al. Heterochromatin-driven nuclear softening protects the genome against mechanical stress-induced damage. Cell. 2020;181:800-17.

53. Makhija E, Jokhun DS, Shivashankar GV. Nuclear deformability and telomere dynamics are regulated by cell geometric constraints. Proc Natl Acad Sci USA. 2016;113:E32-40.

54. Pfeifer CR, Xia Y, Zhu K, Liu D, Irianto J, García VMM, et al. Constricted migration increases DNA damage and independently represses cell cycle. Mol Biol Cell. 2018;29:1948-62.

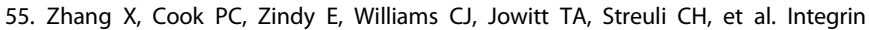
alpha4beta1 controls G9a activity that regulates epigenetic changes and nuclear properties required for lymphocyte migration. Nucleic Acids Res. 2016;44:3031-44.

56. Gerlitz G, Bustin M. Efficient cell migration requires global chromatin condensation. J Cell Sci. 2010;123:2207-17.

57. Madrazo E, Ruano D, Abad L, Alonso-Gómez E, Sánchez-Valdepeñas C, GonzálezMurillo A. et al. G9a Correlates with VLA-4 integrin and influences the migration of childhood acute lymphoblastic leukemia cells. Cancers (Basel). 2018;10:325

58. Sison EA, Magoon D, Li L, Annesley CE, Romagnoli B, Douglas GJ, et al. POL5551, a novel and potent CXCR4 antagonist, enhances sensitivity to chemotherapy in pediatric ALL. Oncotarget. 2015;6:30902-18.

59. Randhawa S, Cho BS, Ghosh D, Sivina M, Koehrer S, Müschen M, et al. Effects of pharmacological and genetic disruption of CXCR4 chemokine receptor function in B-cell acute lymphoblastic leukaemia. $\mathrm{Br} J$ Haematol. 2016;174:425-36.

60. Barbieri F, Bajetto A, Thellung S, Würth R, Florio T. Drug design strategies focusing on the CXCR4/CXCR7/CXCL12 pathway in leukemia and lymphoma. Expert Opin Drug Disco. 2016;11:1093-109.

61. Redondo-Muñoz J, García-Pardo A, Teixidó J. Molecular players in hematologic tumor cell trafficking. Front Immunol. 2019;10:156.

62. San José-Enériz E, Agirre X, Rabal O, Vilas-Zornoza A, Sanchez-Arias JA, Miranda $E$, et al. Discovery of first-in-class reversible dual small molecule inhibitors against G9a and DNMTs in hematological malignancies. Nat Commun. 2017;8:15424.

63. Dong C, Wu Y, Yao J, Wang Y, Yu Y, Rychahou PG, et al. G9a interacts with Snail and is critical for Snail-mediated E-cadherin repression in human breast cancer. J Clin Invest. 2012;122:1469-86.

64. Yokoyama M, Chiba T, Zen $Y$, Oshima M, Kusakabe $Y$, Noguchi $Y$, et al. Histone lysine methyltransferase G9a is a novel epigenetic target for the treatment of hepatocellular carcinoma. Oncotarget. 2017;8:21315-26.

65. Isham CR, Tibodeau JD, Jin W, Xu R, Timm MM, Bible KC. Chaetocin: a promising new antimyeloma agent with in vitro and in vivo activity mediated via imposition of oxidative stress. Blood. 2007;109:2579-88. 
66. Jung HJ, Seo I, Casciello F, Jacquelin S, Lane SW, Suh SI, et al. The anticancer effect of chaetocin is enhanced by inhibition of autophagy. Cell Death Dis. 2016;7: e2098.

\section{ACKNOWLEDGEMENTS}

The authors thank the Microscopy Unit and the Flow Cytometry Core Unit of Instituto de Investigación Biosanitaria Gregorio Marañón (liSGM) for assistance with confocal, videomicroscopy and flow cytometry analyses. The UCM-Genomic CAI Unit for their assistance with the microarray experiments. We thank Dr. Ignacio Casal, Joaquín Teixidó, Alicia García-Arroyo and María Montoya for insightful and critical comments. This research was supported by a FPI Scholarship 2018 (Ministerio de Ciencia e Innovacion/MICINN Agencia Estatal de Investigacion/AEl y Fondo Europeo de Desarrollo Regional/FEDER) to $R$ G.N.; an undergraduate fellowship "Beca de Introducción a la Investigación" (Asociación Española Contra el Cáncer) to C.O.P.; and by grants from Asociación Pablo Ugarte to M.R. Gilead Sciences International Scholar in Hematology/Oncology (Gilead), 2020 Leonardo Grant for Researchers and Cultural Creators (BBVA Foundation) and SAF2017-86327-R (Ministerio de Ciencia e Innovacion/MICINN, Agencia Estatal de Investigacion/AEl y Fondo Europeo de Desarrollo Regional/FEDER) to J.R.M.

\section{AUTHOR CONTRIBUTIONS}

EM carried out biochemical and imaging experiments and contributed to the interpretation of biological data. RGN performed biochemical and in vivo studies.
COP contributed to biochemical and imaging experiments. AGM provided clinically annotated multiple ALL samples and contributed to in vivo experiments and the interpretation of clinical data. MGL carried out the bioinformatics analysis of ChIP-seq and microarray data samples and contributed to the interpretation of data. MR designed part of the studies, provided ALL samples, and contributed to the interpretation of clinical data. JRM designed the studies, supervised the project, and wrote the manuscript with the input of all authors.

\section{COMPETING INTERESTS}

The authors declare no competing interests.

\section{ADDITIONAL INFORMATION}

Supplementary information The online version contains supplementary material available at https://doi.org/10.1038/s41388-021-02168-8.

Correspondence and requests for materials should be addressed to Javier RedondoMuñoz.

Reprints and permission information is available at http://www.nature.com/ reprints

Publisher's note Springer Nature remains neutral with regard to jurisdictional claims in published maps and institutional affiliations. 